\title{
Introduction to the Fractional Fourier Transform and Its Applications
}

\author{
Haldun M. Ozaktas and M. Alper Kutay \\ Department of Electrical Engineering \\ Bilkent University \\ TR-06533 Bilkent, Ankara, Turkey
}

David Mendlovic

Faculty of Engineering, Tel-Aviv University

69978 Tel-Aviv, Israel

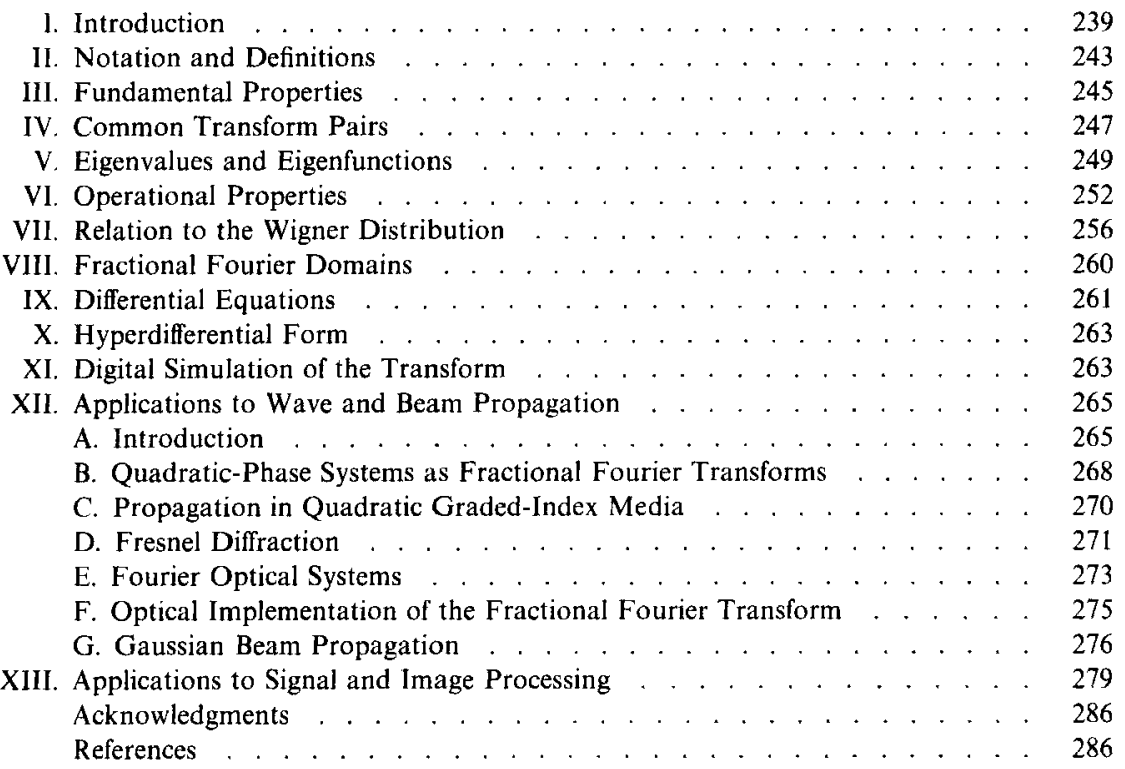

\section{INTRODUCTION}

The purpose of this chapter is to provide a self-complete introduction to the fractional Fourier transform for those who wish to obtain an understanding of the essentials without having to work through the hundreds of papers 
which have appeared in the last few years. A general introduction will be followed by the definition of the transform and a discussion of its fundamental and operational properties. Of central importance is the relationship of the transform to the Wigner distribution and other phase-space distributions (also known as time-frequency or space-frequency representations). We will concentrate on two main application areas which have so far received the most attention: wave and beam propagation and signal processing.

The fractional Fourier transform is a generalization of the ordinary Fourier transform with an order parameter $a$. Mathematically, the $a$ th order fractional Fourier transform is the ath power of the Fourier transform operator. The $a=1$ st order fractional transform is the ordinary Fourier transform. With the development of the fractional Fourier transform and related concepts, we see that the ordinary frequency domain is merely a special case of a continuum of fractional Fourier domains, and we arrive at a richer and more general theory of alternate signal representations, all of which are elegantly related to phase-space distributions. Every property and application of the common Fourier transform becomes a special case of that of the fractional transform. In every area in which Fourier transforms and frequency domain concepts are used, there exists the potential for generalization and improvement by using the fractional transform. For instance, the well-known result stating that the far-field diffraction pattern of an aperture is in the form of the Fourier transform of the aperture can be generalized to state that at closer distances, one observes the fractional Fourier transform of the aperture. The theory of optimal Wiener filtering in the ordinary Fourier domains can be generalized to optimal filtering in fractional domains, resulting in smaller mean-square errors at practically no additional cost.

In essence, the ath order fractional Fourier transform interpolates between a function $f(u)$ and its Fourier transform $F(\mu)$. The 0th order transform is simply the function itself, whereas the 1st order transform is its Fourier transform. The 0.5 th transform is something in between, such that the same operation that takes us from the original function to its 0.5 th transform will take us from its 0.5 th transform to its ordinary Fourier transform. More generally, index additivity is satisfied: The $a_{2}$ th transform of the $a_{1}$ th transform is equal to the $\left(a_{2}+a_{1}\right)$ th transform. The -1 th transform is the inverse Fourier transform, and the - ath transform is the inverse of the ath transform.

Scattered early papers related to the fractional Fourier transform include Wiener [1929], Condon [1937], Bargmann [1961], and de Bruijn [1973]. Of importance are two separate streams of mathematical papers which appeared throughout the eighties [Namias, 1980; McBride and Kerr, 1987; 
Mustard, 1987a,b, 1989, 1991, 1996]. However, the number of publications exploded only after the introduction of the transform to the optics and signal processing communities [Seger, 1993; Lohmann, 1993; Ozaktas and Mendlovic, 1993a,b; Mendlovic and Ozaktas, 1993; Ozaktas and others, 1994a; Alieva and others, 1994; Almeida, 1994]. Not all of these authors were aware of each other or building on the work of those preceding them, nor is the transform always immediately recognizable in some of these works.

The fractional Fourier transform (or essentially equivalent transforms) appears in many contexts, although it has not always been recognized as being the fractional power of the Fourier transform and thus referred to as the fractional Fourier transform. For instance, the Green's function of the quantum-mechanical harmonic oscillator is the kernel of the fractional Fourier transform. Also, the fractional Fourier transform is a special case of the more general linear canonical transform (see Wolf [1979] for an introduction and references). This transform has been studied in many contexts, but again the particular special case which is the fractional Fourier transform has usually not been recognized as such.

The preceding citations do not represent a complete list of known historical references. For a more complete list and also a more comprehensive treatment of the fractional Fourier transform and its relation to phase-space distributions, we refer the reader to a forthcoming book on the subject by the authors (Wiley, to be publ. 1999). We expect further scattered historical references not known to us to be revealed in time. Given the multitude of contexts in which essentially equivalent or closely related integral transforms appear, it is probably not possible to attribute its invention to a particular set of authors. These many contexts in which it was reinvented time after time in different guises is testimony to the elegance and ubiquity of the transform.

Given the widespread use of the ordinary Fourier transform in science and engineering, it is important to recognize this integral transform as the fractional power of the Fourier transform. Indeed, it has been this recognition which has inspired most of the many recent applications. Replacing the ordinary Fourier transform with the fractional Fourier transform (which is more general and includes the ordinary Fourier transforms as its special case) adds an additional degree of freedom to the problem, represented by the order parameter $a$. This in turn may allow either a more general formulation of the problem (as in the diffraction from an aperture example) or improvements based on the possibility of optimizing over $a$ (as in the optimal Wiener filtering example).

The fractional Fourier transforms has been found to have several applications in the area known as analog optical information processing, or 
Fourier optics. This transform allows a reformulation of this area in a way much more general than that found in standard texts on the subject. It has also led to generalizations of the notions of space (or time) and frequency domains, which are central concepts in signal processing, leading to many applications in this area. More generally, the transform may be expected to have an impact in the form of deeper understanding or new applications in every area in which the Fourier transform plays a significant role, and to take its place among the standard mathematical tools of physics and engineering.

More specifically, some applications which have already been investigated or suggested include diffraction theory [Alieva and others, 1994; Gori, Santarsiero, and Bagini, 1994; Pellat-Finet, 1994; Pellat-Finet, 1995; Ozaktas and Mendlovic, 1995; Abe and Sheridan, 1995a; Alonso and Forbes, 1997; Ozaktas and Erden, 1997], optical beam propagation and spherical mirror resonators (lasers) [Ozaktas and Mendlovic, 1994; Erden and Ozaktas, 1997; Ozaktas and Erden, 1997], propagation in graded index media [Ozaktas and Mendlovic, 1993a,b; Mendlovic and Ozaktas, 1993; Mendlovic, Ozaktas, and Lohmann, 1994a; Alieva and Agulló-López, 1995; Abe and Sheridan, 1995b; Gómez-Reino, Bao, and Pérez, 1996], Fourier optics [Bernardo and Soares, 1994a,b; Pellat-Finet and Bonnet, 1994; Ozaktas and Mendlovic, 1995; Ozaktas and Mendlovic, 1996], statistical optics [Erden, Ozaktas, and Mendlovic, 1996a,b], optical systems design [Dorsch, 1995; Dorsch and Lohmann, 1995; Lohmann, 1995], quantum optics [Yurke and others, 1990; Aytür and Ozaktas, 1995], radar and phase retrieval [Raymer, Beck, and McAlister, 1994a,b; McAlister and others, 1995], tomography [Beck and others, 1993; Smithey and others, 1993; Lohmann and Soffer, 1994; Wood and Barry, 1994a,b], signal detection, correlation, and pattern recognition [Mendlovic, Ozaktas, and Lohmann, 1995d; Alieva and Agulló-López, 1995; Garcia and others, 1996; Lohmann, Zalevsky, and Mendlovic, 1996b; Bitran and others, 1996; Mendlovic and others, 1995a; Mendlovic, Zalevsky, and Ozaktas, 1998], space- or timevariant filtering [Ozaktas and others, 1994a; Granieri, Trabocchi, and Sicre, 1995; Mendlovic and others, 1996b; Ozaktas, 1996; Zalevsky and Mendlovic, 1996; Mendlovic and others, 1996b; Kutay and others, 1997; Mustard, 1997], signal recovery, restoration, and enhancement [Lohmann and others, 1996a; Erden and others, 1997a,b; Ozaktas, Erden, and Kutay, 1997; Kutay and Ozaktas, 1998; Kutay and others, 1998a,b], multiplexing and data compression [Ozaktas and others, 1994a], study of space- or timefrequency distributions [Almeida, 1994; Fonollosa and Nikias, 1994; Lohmann and Soffer, 1994; Ozaktas and others, 1994a; Mendlovic and others, 1995c; Dragoman, 1996; Mendlovic and others, 1996a; Ozaktas, Erkaya, and Kutay, 1996a; Mihovilovic and Bracewell, 1991], and solution of 
differential equations [Namias, 1980; McBride and Kerr, 1987]. We believe that these are only a fraction of the possible applications. We hope that this chapter will make possible the discovery of new applications by introducing the subject to new audiences.

\section{Notation and Definitions}

The ath order fractional Fourier transform of the function $f(u)$ will most often be denoted by $f_{a}(u)$ or, equivalently, $\mathscr{F} a f(u)$. When there is possibility of confusion, we may more explicitly write $\mathscr{F}^{a}[f(u)]$. The transform is defined as a linear integral transform with kernel $K_{a}\left(u, u^{\prime}\right)$ :

$$
f_{a}(u)=\mathscr{F}^{a}[f(u)]=\int K_{a}\left(u, u^{\prime}\right) f\left(u^{\prime}\right) d u^{\prime} .
$$

The kernel will be given explicitly in the following text. All integrals are from minus to plus infinity unless otherwise stated. We prefer to use the same dummy variable $u$ both for the original function in the space (or time) domain and its fractional Fourier transform. This is in contrast to the conventional practice associated with the ordinary Fourier transform, where a different symbol, say $\mu$, denotes the argument of the Fourier transform $F(\mu)$ :

$$
\begin{aligned}
& F(\mu)=\int f(u) e^{-i 2 \pi \mu u} d u, \\
& f(u)=\int F(\mu) e^{i 2 \pi \mu u} d \mu .
\end{aligned}
$$

But these can be rewritten as

$$
\begin{aligned}
& F(u)=\int f\left(u^{\prime}\right) e^{-i 2 \pi u u^{\prime}} d u^{\prime} \\
& f(u)=\int F\left(u^{\prime}\right) e^{i 2 \pi u^{\prime} u} d \mu^{\prime} .
\end{aligned}
$$

When it is desirable to distinguish the argument of the transformed function from that of the original function, we will let $u_{a}$ denote the argument of the ath order fractional Fourier transform: $f_{a}\left(u_{a}\right)=\left(\mathscr{F}^{a}[f(u)]\right)\left(u_{a}\right)$. With this convention, $u_{0}$ corresponds to $u$, the space (or time) coordinate; $u_{1}$ corresponds to the spatial (or temporal) frequency coordinate $\mu$; and $u_{2}=-u_{0}$, $u_{3}=-u_{1}$. Finally, we will agree to always interpret $u$ as a dimensionless variable. 
We will refer to $\mathscr{F}^{a}[\cdot]$, or simply $\mathscr{F}^{a}$, as the ath order fractional Fourier transform operator. This operator transforms a function $f(u)$ into its fractional Fourier transform $f_{a}(u)$. We will restrict ourselves to the case where the order parameter $a$ is a real number. The signal $f$ is a finite energy signal and $f(u)$ is a finite energy function both of which are well behaved in the sense usually presumed in physical applications. In quantum mechanics $f$ is the abstract state vector $|f\rangle$ and $f(u)=\langle u \mid f\rangle$ is the $u$-representation of $f$. Likewise, $f_{a}(u)=\left\langle u_{a} \mid f\right\rangle$ is the $u_{a}$-representation, which we will also refer to as the representation of $f$ in the ath order fractional Fourier domain. In this context $|f(u)|^{2}$ is interpreted as a probability distribution so that the energy of the function $\operatorname{En}[f]=f|f(u)|^{2} d u=\langle f \mid f\rangle$ corresponds to its integrated probability and is thus equal to 1 . In signal processing and optics, the energy can take on any finite value but is conserved if attenuation or amplification mechanisms do not exist. (We will also deal with sets of signals and functions whose energies are not finite (delta functions and harmonic functions); these will not correspond to physically realizable functions, but rather serve as intermediaries in our formulations.)

We now define the ath order fractional Fourier transform $f_{a}(u)$ through the following linear integral transform:

$$
\begin{aligned}
f_{a}(u) & =\int K_{a}\left(u, u^{\prime}\right) f\left(u^{\prime}\right) d u^{\prime}, \\
K_{a}\left(u, u^{\prime}\right) & =A_{\phi} \exp \left[i \pi\left(\cot \phi u^{2}-2 \csc \phi u u^{\prime}+\cot \phi u^{\prime 2}\right)\right] .
\end{aligned}
$$

where

$$
\begin{aligned}
\phi & =\frac{a \pi}{2}, \\
A_{\phi} & =\sqrt{1-i \cot \phi} .
\end{aligned}
$$

The square root is defined such that the argument of the result lies in the interval $(-\pi / 2, \pi / 2]$. The kernel is not strictly defined when $a$ is an even integer. However, it is possible to show that as $a$ approaches an even integer, the kernel behaves like a delta function under the integral sign. Thus, consistent with the limiting behavior of the above kernel for values of $a$ approaching even integers (further discussed later), we define $K_{4 j}\left(u, u^{\prime}\right)$ $=\delta\left(u-u^{\prime}\right)$ and $K_{4 j \pm 2}\left(u, u^{\prime}\right)=\delta\left(u+u^{\prime}\right)$, where $j$ is an arbitrary integer. Generally speaking, the fractional Fourier transform of $f(u)$ exists under the same conditions under which its Fourier transform exists [McBride and Kerr, 1987; Almeida, 1994]. 


\section{Fundamental Properties}

We first examine the case when $a$ is equal to an integer $j$. We note that by definition $\mathscr{F}^{4 j}$ and $\mathscr{F}^{4 j \pm 2}$ correspond to the identity operator $\mathscr{Z}$ and the parity operator $\mathscr{P}$, respectively (that is, $f_{4 j}(u)=f(u)$ and $f_{4 j \pm 2}(u)=$ $f(-u)$ ). For $a=1$ we find $\phi=\pi / 2, A_{\phi}=1$, and

$$
f_{1}(u)=\int_{-\infty}^{\infty} \exp \left(-i 2 \pi u u^{\prime}\right) f\left(u^{\prime}\right) d u^{\prime} .
$$

We see that $f_{1}(u)$ is equal to the ordinary Fourier transform of $f(u)$, which was previously denoted by the conventional upper case $F(u)$. Likewise, it is possible to see that $F_{-1}(u)$ is the ordinary inverse Fourier transform of $f(u)$. Our definition of the fractional Fourier transform is consistent with defining integer powers of the Fourier transform through repeated application (that is, $\mathscr{\mathscr { F }}^{2}=\mathscr{\mathscr { F }} \mathscr{F}, \mathscr{F}^{3}=\mathscr{F}^{2} \mathscr{F}^{2}$, and so on). Since $\phi=a \pi / 2$ appears in Equation 6 only in the argument of trigonometric functions, the definition is periodic in $a$ (or $\phi$ ) with period 4 (or $2 \pi$ ). Thus it is sufficient to limit attention to the interval $a \in[-2,2)$. These facts can be restated in operator notation:

$$
\begin{aligned}
\mathscr{F}^{0} & =\mathscr{Z}, \\
\mathscr{F}^{1} & =\mathscr{F}, \\
\mathscr{F}^{2} & =\mathscr{P}, \\
\mathscr{F}^{3} & =\mathscr{F} \mathscr{P}=\mathscr{P} \mathscr{F}, \\
\mathscr{F}^{4} & =\mathscr{F} 0=\mathscr{Z}, \\
\mathscr{F}^{4 j+a} & =\mathscr{F}^{4 j^{\prime}+a},
\end{aligned}
$$

where $j, j^{\prime}$ are arbitrary integers.

Let us now examine the behavior of the kernel for small $|a|>0$ :

$$
K_{a}\left(u, u^{\prime}\right)=\frac{e^{-i \pi \mathrm{sgn}(\phi) / 4}}{\sqrt{|\phi|}} \exp \left[i \pi\left(u-u^{\prime}\right)^{2} / \phi\right] .
$$

Now, using the well-known limit

$$
\delta(u)=\lim _{c \rightarrow 0} e^{-i \pi / 4} \sqrt{\frac{1}{c}} e^{i \pi u^{2} / c},
$$

the kernel is seen to approach $\delta\left(u-u^{\prime}\right)$ as $a$ approaches 0 . Thus defining the kernel $K_{a}\left(u, u^{\prime}\right)$ to be precisely $\delta\left(u-u^{\prime}\right)$ at $a=0$ maintains continuity of the transform with respect to $a$. A similar discussion is possible when $a$ 
approaches other integer multiples of 2. A more rigorous discussion of continuity with respect to $a$ may be found in McBride and Kerr [1987].

We now discuss the index additivity property:

$$
\mathscr{F}^{a_{1}} \mathscr{F}^{a_{2}} f(u)=\mathscr{F}^{a_{1}+a_{2}} f(u)=\mathscr{F}^{a_{2}} \mathscr{F}^{a_{1}} f(u),
$$

or in operator notation

$$
\mathscr{F}^{a_{1}} \mathscr{F}^{a_{2}}=\mathscr{F}^{a_{1}+a_{2}}=\mathscr{F}^{a_{2}} \mathscr{F}^{a_{1}} .
$$

This can be proved by repeated application of Equation 6, and amounts to showing

$$
\int K_{a_{2}}\left(u, u^{\prime \prime}\right) K_{a_{1}}\left(u^{\prime \prime}, u^{\prime}\right) d u^{\prime \prime}=K_{a_{1}+a_{2}}\left(u, u^{\prime}\right)
$$

by direct integration, which can be accomplished by using standard Gaussian integrals. We do not present the details of this proof, since this property will follow much more simply from certain properties of the transform to be discussed.

The index additivity property is of central importance. Indeed, without it, we could hardly think of $\mathscr{F}^{a}$ as being the ath power of $\mathscr{F}$ (more will be said on this later). For instance, the 0.2nd fractional Fourier transform of the 0.5 th transform is the 0.7 th Fourier transform. Repeated application leads to statements such as, for instance, the 1.3th transform of the 2.1st transform of the 1.4th transform is the 4.8th transform (which is the same as the 0.8 th transform). Transforms of different orders commute with each other so that their order can be freely interchanged. From the index additivity property, we deduce that the inverse of the ath order fractional Fourier transform operator $\left(\mathscr{F}^{a}\right)^{-1}$ is simply equal to the operator $\mathscr{F}^{-a}$ (because $\mathscr{F}^{-a} \mathscr{F}^{a}=\mathscr{Z}$ ). This can also be shown by directly demonstrating that

$$
\int K_{a}\left(u, u^{\prime \prime}\right) K_{-a}\left(u^{\prime \prime}, u^{\prime}\right) d u=\delta\left(u-u^{\prime}\right),
$$

so that $K_{a}^{-1}\left(u, u^{\prime}\right)=K_{-a}\left(u, u^{\prime}\right)$. Thus we see that we can freely manipulate the order parameter $a$ as if it denoted a power of the Fourier transform operator $\mathscr{\mathscr { F }}$.

Fractional Fourier transforms constitute a one-parameter family of transforms. This family is a subfamily of the more general family of linear canonical transforms which have three parameters [Wolf, 1979; Mohinsky and Quesne, 1971; and Mohinsky, Seligman, and Wolf, 1972]. As all linear canonical transforms do, fractional Fourier transforms satisfy the associativity property and they are unitary, as we can directly see by examining the 
kernel of the inverse transform obtained by replacing $a$ with $-a$ :

$$
K_{a}^{-1}\left(u, u^{\prime}\right)=K_{-a}\left(u, u^{\prime}\right)=K_{a}^{*}\left(u, u^{\prime}\right)=K_{a}^{*}\left(u^{\prime}, u\right) .
$$

The kernel $K_{a}\left(u, u^{\prime}\right)$ is symmetric and unitary, but not Hermitian. Unitarity implies that the fractional Fourier transform can be interpreted as a transformation from one representation to another, and that inner products and norms are not changed under the transformation.

\section{Common Transform Pairs}

Table 1 gives the fractional Fourier transforms of a number of functions for which the integral appearing in Equation 6 can be evaluated analytically (often using standard Gaussian integrals). More will be said on the fractional Fourier transforms of chirp functions $\exp \left[i \pi\left(\chi u^{2}+2 \xi u\right)\right]$ after we discuss the Wigner rotation property of the transform.

Greater insight can be obtained by considering some numerically obtained illustrations. Indeed, the fractional Fourier transforms of many common functions do not have simple closed-form expressions. These may be obtained numerically using the algorithm discussed in Section 11 later. We know that when $a=0$ we have the original function, and when $a=1$ we have its ordinary Fourier transform. As a varies from 0 to 1 , the transform evolves smoothly from the original function to the ordinary Fourier transform. Figures 1 and 2 show the evolution of the $\operatorname{rect}(u)$

TABLE 1

The Functions on the Right are the Fractional Fourier Transforms of the Functions on the LefT; $j$ is an ARBitrary Integer, and $\xi$ and $\chi$ are Real Constants. For Certain Isolated Values of $a$, the Expressions Below Should be Inter preted in the Limiting Sense (Equation 17). In the Last Pair, $\chi>0$ is Required for Convergence.

\begin{tabular}{|c|c|}
\hline$f(u)$ & $f_{a}(u)$ \\
\hline$\delta(u-\xi)$ & $A_{\phi} \exp \left[i \pi\left(u^{2} \cot \phi-2 u \xi \csc \phi+\xi^{2} \cot \phi\right)\right]$ \\
\hline $\exp (i 2 \pi \xi u)$ & $\sqrt{1+i \tan \phi} \exp \left[-i \pi\left(u^{2} \tan \phi-2 u \xi \sec \phi+\xi^{2} \tan \phi\right)\right]$ \\
\hline $\exp \left(i \pi \gamma u^{2}\right)$ & $\sqrt{(1+i \tan \phi) /(1+\chi \tan \phi)} \exp \left[i \pi u^{2}(\chi-\tan \phi) /(1+\chi \tan \phi)\right]$ \\
\hline $\exp \left[i \pi\left(\chi u^{2}+2 \xi u\right)\right.$ & $\begin{array}{l}\sqrt{(1+i \tan \phi) /(1+\chi \tan \phi)} \exp \left\{i \pi\left[u^{2}(\chi-\tan \phi)\right.\right. \\
\left.\left.+2 u \xi \sec \phi-\xi^{2} \tan \phi\right] /[1+\chi \tan \phi]\right\}\end{array}$ \\
\hline $\exp \left(-\pi x u^{2}\right)$ & $\begin{aligned} \sqrt{(1-i \cot \phi) /(\chi-i \cot \phi)} & \exp \left[i \pi u^{2} \cot \phi\left(\chi^{2}-1\right) /\left(\chi^{2}+\cot ^{2} \theta\right)\right] \\
& \times \exp \left[-\pi u^{2} \chi \csc ^{2} \phi /\left(\chi^{2}+\cot ^{2} \phi\right)\right]\end{aligned}$ \\
\hline
\end{tabular}




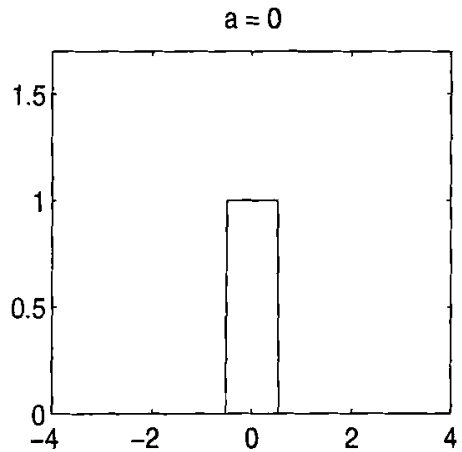

(a)

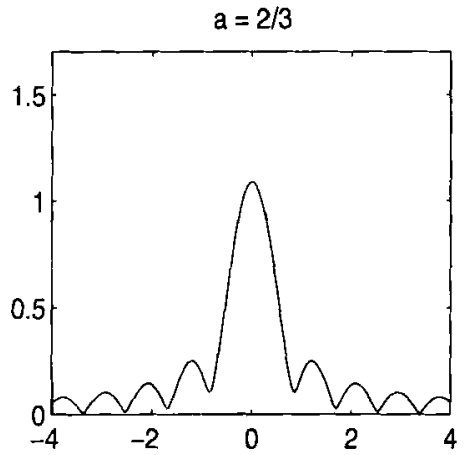

(c)

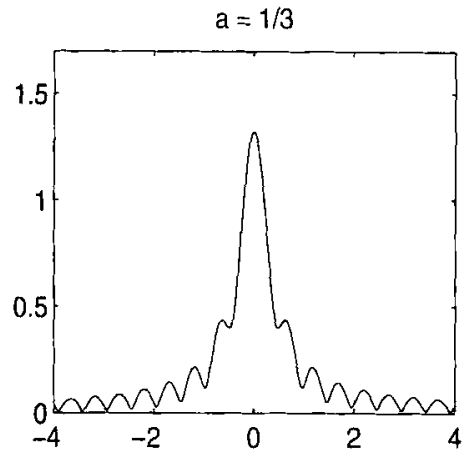

(b)

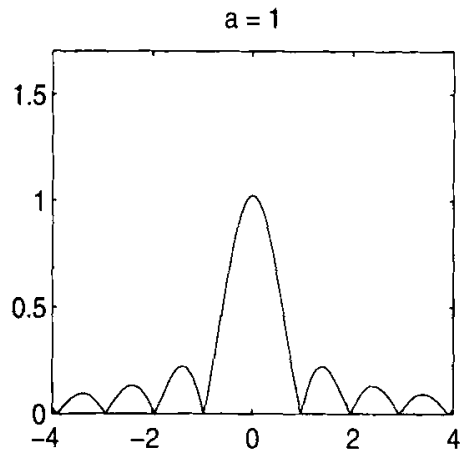

(d)

Figure 1. Magnitudes of the fractional Fourier transforms of the rectangle function, I.

function into the $\operatorname{sinc}(u) \equiv(\sin \pi u) /(\pi u)$ function. Figure 3 shows the real parts of the fractional Fourier transforms of the Dirac delta function $\delta(u-1)$. We note that for orders close to zero, the transform of the delta function is highly oscillatory, and thus will approximately behave like the delta function under the integral sign, averaging out to zero whatever function it happens to multiply.

Finally, we give the fractional Fourier transform of the quadratic phase function $f(u)=\exp (-i \pi / 4) \sqrt{1 / r_{c}} \exp \left(i \pi u^{2} / r_{c}\right)$ with complex radius $r_{c}$ :

$$
f_{a}(u)=\sqrt{\frac{1-i \cot \phi}{1+r_{c} \cot \phi}} e^{i \pi u^{2}\left[1-r_{c} \tan \phi i r_{c}+\tan \phi\right]}
$$

provided $\mathscr{I}\left(r_{c}\right) \leqslant 0$, which is also the condition for the original function $f(u)$ to have finite energy. From this result we conclude that the complex radius 


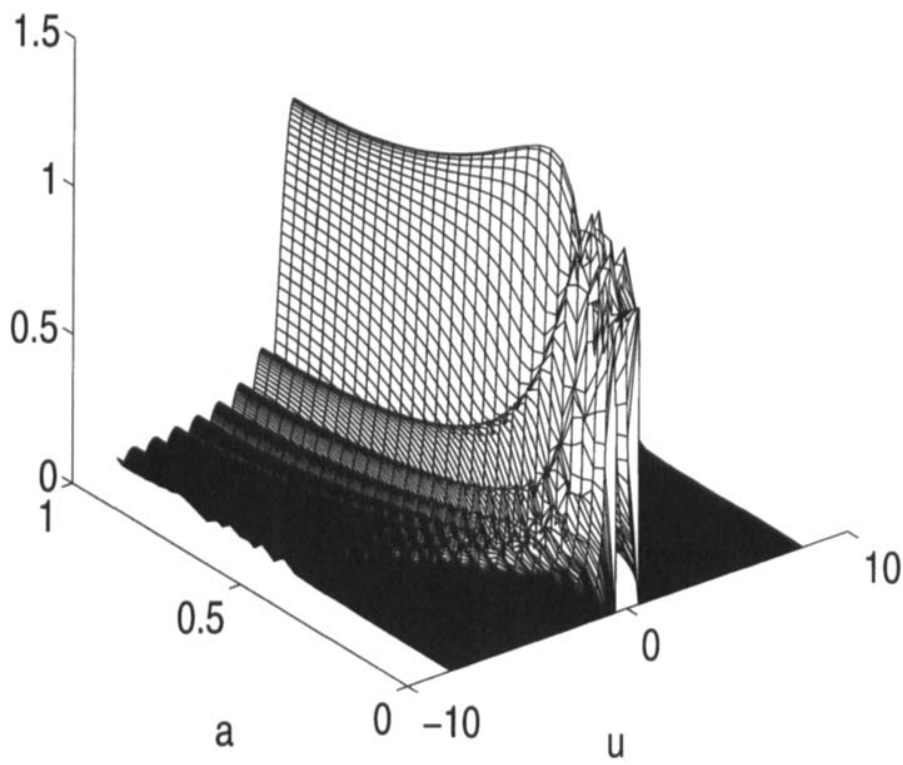

FIGURE 2. Magnitudes of the fractional Fourier transforms of the rectangle function, II.

$r_{c}^{\prime}$ of the transformed function is

$$
r_{c}^{\prime}=\frac{r_{c}+\tan \phi}{1-r_{c} \tan \phi} .
$$

This result is useful in beam propagation problems since the original function $f(u)$ represents a Gaussian beam with complex radius $r_{c}$.

\section{Eigenvalues and Eigenfunctions}

The eigenvalues and eigenfunctions of the ordinary Fourier transform are well known (although seldom discussed in introductory texts). They are the Hermite-Gaussian functions $\psi_{n}(u)$, commonly known as the eigensolutions of the harmonic oscillator in quantum mechanics, or the modes of propagation of quadratic graded-index media in optics. The eigenvalues may be expressed as $\exp (-i n \pi / 2)$ and are given by $1,-i,-1, i, 1,-i, \ldots$ for $n=0$, $1,2,3,4,5, \ldots$ Thus the eigenvalue equation for the ordinary Fourier transform may be written as

$$
\mathscr{F} \psi_{n}(u)=e^{-i n \pi / 2} \psi_{n}(u),
$$




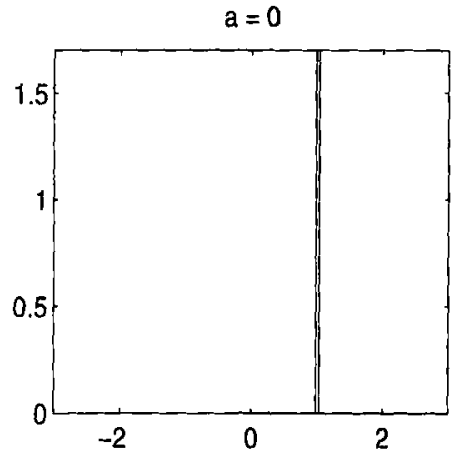

(a)

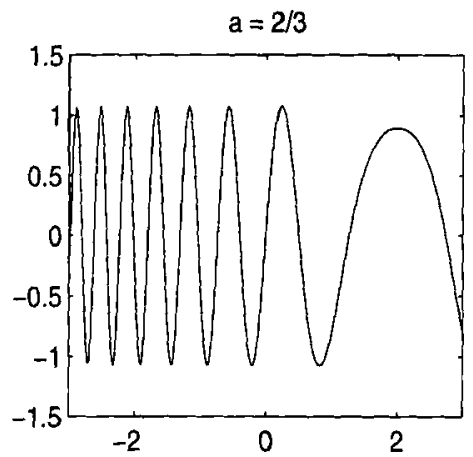

(c)

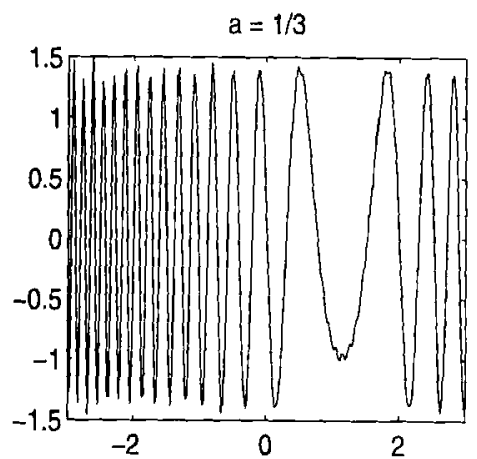

(b)

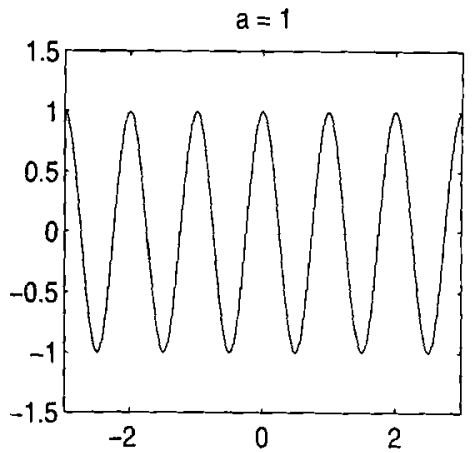

(d)

FIGURE 3. Real parts of the fractional Fourier transforms of a delta function.

where the Hermite-Gaussian functions are more explicitly given by

$$
\begin{gathered}
\psi_{n}(u)=A_{n} H_{n}(\sqrt{2 \pi} u) e^{-\pi n^{2}}, \\
A_{n}=2^{1 / 4} / \sqrt{2^{n} n !},
\end{gathered}
$$

for $n=0,1,2,3,4,5, \ldots$ Here $H_{n}(u)$ are the Hermite polynomials. The particular scale factors which appear in this equation are a direct consequence of the way we have defined the Fourier transform with $2 \pi$ in the exponent.

The ath order fractional Fourier transform shares the same eigenfunctions as the Fourier transform, but its eigenvalues are the ath power of the eigenvalues of the ordinary Fourier transform:

$$
\mathscr{F}^{a} \psi_{n}(u)=e^{-i a n \pi / 2} \psi_{n}(u)
$$


This result can be established directly from Equation 6 by induction. First, we can show that $\psi_{0}(u)$ and $\psi_{1}(u)$ are eigenfunctions with eigenvalues 1 and $\exp (-i a \pi / 2)$ by evaluating the resulting standard complex exponential integrals. Then, by using standard recurrence relations for the HermiteGaussian functions it is possible to assume that the result-to-be-shown holds for $n-1$ and $n$, and show that as a consequence it holds for $n+1$. This completes the induction.

The preceding demonstrated outline of the fact that Hermite-Gaussian functions are eigenfunctions of the fractional Fourier transform as defined by Equation 6 then reduces to the well-known fact that Hermite-Gaussian functions are eigenfunctions of the ordinary Fourier transform when $a=1$, since Equation 6 reduces to the definition of the ordinary Fourier transform and since $e^{-i a n \pi / 2}$ reduces to $e^{-i n \pi / 2}$ when $a=1$.

Readers familiar with functions $F N(\mathscr{A})$ of an operator (or matrix) $\mathscr{A}$ with eigenvalues $\lambda_{n}$ will know that in general $F N(\mathscr{A})$ will have the same eigenfunctions as $\mathscr{A}$ and that its eigenvalues will be $F N\left(\lambda_{n}\right)$. The above eigenvalue equation is particularly satisfying in this light since $\mathscr{F}^{a}$, as we have defined it, is indeed seen to correspond to the ath power of the Fourier transform operator $\left(F N(\cdot)=(\cdot)^{a}\right)$. However, it should be noted that the definition of the ath power function is ambiguous, and our definition of the fractional Fourier transform through Equation 6 is associated with a particular way of resolving the ambiguity associated with the ath power function (Equation 28). Other definitions of the transform also deserving to be called the fractional power of the Fourier transform are possible. The particular definition we are considering is the one that has been most studied and that has led to the greatest number of interesting applications. We are convinced it has a special place among other possible definitions.

Knowledge of the complete set of eigenvalues and eigenfunctions of a linear operator is sufficient to completely characterize the operator. In fact, in some works the fractional Fourier transform has been defined through its eigenvalue equation [Namias, 1980; Ozaktas and Mendlovic, 1993a,b; Mendlovic and Ozaktas, 1993]. To find the fractional transform of a given function $f(u)$ from knowledge of the eigenfunctions and eigenvalues only, we first expand the function as a linear superposition of the eigenfunctions of the fractional Fourier transform (which are known to constitute a complete set):

$$
\begin{aligned}
f(u) & =\sum_{n=0}^{\infty} C_{n} \psi_{n}(u), \\
C_{n} & =\int \psi_{n}(u) f(u) d u .
\end{aligned}
$$


Applying $\mathscr{F}^{a}$ on both sides of Equation 29 and using Equation 28, one obtains

$\mathscr{F}^{a} f(u)=\sum_{n=0}^{\infty} e^{-i a n \pi / 2} C_{n} \psi_{n}(u)=\int \sum_{n=0}^{\infty} e^{-i a n \pi / 2} \psi_{n}(u) \psi_{n}\left(u^{\prime}\right) f\left(u^{\prime}\right) d u^{\prime}$.

Upon comparison with Equation 6, the kernel $K_{a}\left(u, u^{\prime}\right)$ is identified as

$$
K_{a}\left(u, u^{\prime}\right)=\sum_{n=0}^{\infty} e^{-i a n \pi / 2} \psi_{n}(u) \psi_{n}\left(u^{\prime}\right) .
$$

This is the spectral decomposition of the kernel of the fractional Fourier transform. The kernel given in Equation 32 can be shown to be identical to that given in Equation 6 directly by using an identity known as Mehler's formula:

$$
\sum_{n=0}^{\infty} \frac{e^{i n \phi}}{2^{n} n ! \sqrt{\pi}} H_{n}(u) H_{n}\left(u^{\prime}\right)=\frac{1}{\sqrt{\pi} \sqrt{1-\exp (2 i \phi)}} \exp \left[\frac{2 u u^{\prime} e^{i \phi}-e^{2 i \phi}\left(u^{2}+u^{\prime 2}\right)}{1-e^{2 i \phi}}\right] .
$$

Several properties of the fractional Fourier transform immediately follow from Equation 28. In particular the special cases $a=0, a=1$, and the index additivity property are deduced easily. (The latter can be shown by applying $\mathscr{F}^{a^{\prime}}$ to both sides of Equation 28.)

\section{Operational Properties}

Various operational properties of the transform are listed in Table 2 [Namias, 1980; McBride and Kerr, 1987; Mendlovic and Ozaktas, 1993; Almeida, 1994]. Most of these are most readily derived or verified by using Equation 6 or the symmetry properties of the kernel.

Operations satisfying the first property are referred to as even operations, so that the fractional Fourier transform is an even operation. This property also implies

$$
\begin{aligned}
& \mathscr{F}^{a}[\operatorname{Ev} f(u)]=\operatorname{Ev} f_{a}(u), \\
& \mathscr{F}^{a}[\operatorname{Od} f(u)]=\operatorname{Od} f_{a}(u),
\end{aligned}
$$

which in turn imply that the transform of an even function is always even and the transform of an odd function is always odd. Similar facts can be stated in operator form: All even operators, and in particular the frac- 
TABLE 2

Operational Properties of the Fractional Fourier Transform. $\xi$ Is an Arbitrary Real Number, $k$ Is a Real Number $(k \neq 0, \pm \infty)$, AND $n$ Is AN INTEGER; $\phi^{\prime}=\arctan \left(k^{2} \tan \phi\right)$, Where $\phi^{\prime}$ Is Taken to Be in the Same Quadrant as $\phi$.

\begin{tabular}{cc}
$f(u)$ & $f_{a}(u)$ \\
$f(-u)$ & $f_{a}(-u)$ \\
$f(k u)$ & $|k|^{-1} \sqrt{(1-i \cot \phi) /\left(1-i k^{-2} \cot \phi\right) \exp \left[i \pi u^{2} \cot \phi\right.}$ \\
$\times(u-\xi)$ & $\begin{array}{c}\left.\left.\cos \phi^{\prime} / \cos ^{2} \phi\right)\right] f_{a^{\prime}}\left(u \sin \phi^{\prime} / k \sin \phi\right) \\
\exp (i 2 \pi \xi u) f(u) \\
u f(u) \\
(i 2 \pi)^{-1} d f(u) / d u \\
\int_{u_{0}}^{u} f\left(u^{\prime}\right) d u^{\prime}\end{array}$ \\
\hline
\end{tabular}

tional Fourier transform operator, commute with the parity operator $\mathscr{P}$ $\left(\mathscr{F}^{a} \mathscr{P}=\mathscr{P}_{\mathscr{F}} \mathscr{F}^{a}\right)$ and satisfy $\mathscr{F}^{a}=\mathscr{P}_{\mathscr{F}} \mathscr{P}^{\circ}$. The eigenfunctions of even operations can always be chosen to be of definite (even or odd) parity (the Hermite-Gaussian functions satisfy this property).

The second property is the generalization of the ordinary Fourier transform property stating that the Fourier transform of $f(k u)$ is $|k|^{-1} F(\mu / k)$. Notice that the fractional Fourier transform of $f(k u)$ cannot be expressed as a scaled version of $f_{a}(u)$ for the same order $a$. Rather, the fractional Fourier transform of $f(k u)$ turns out to be a scaled and chirp-modulated version of $f_{a^{\prime}}(u)$ where $a^{\prime} \neq a$ is a different order.

Now we turn our attention to the fifth and sixth properties. The fractional Fourier transform of $u f(u)$ is equal to a linear combination of $u f_{a}(u)$ and $d f_{a}(u) / d u$. The coefficients of this linear combination are $\cos \phi$ and $-\sin \phi$. When $a=1$, this reduces to the corresponding ordinary Fourier transform property. Similar comments apply to the fractional Fourier transform of $d f(u) / d u$. The essence of these properties are most easily grasped if we express them in pure operator form. Let us define the coordinate multiplication operator $\mathscr{U}$ and differentiation operator $\mathscr{D}$ through their effects in the space domain

$$
\begin{aligned}
& \mathscr{U} f(u)=u f(u), \\
& \mathscr{D} f(u)=\frac{1}{i 2 \pi} \frac{d f(u)}{d u} .
\end{aligned}
$$

These are simply dimensionless versions of the position and momentum 
operators of quantum mechanics and might have been written as

$$
\begin{aligned}
& \langle u \mid \mathscr{U} f\rangle=u\langle u \mid f\rangle, \\
& \langle u \mid \mathscr{D} f\rangle=\frac{1}{i 2 \pi} \frac{d}{d u}\langle u \mid f\rangle .
\end{aligned}
$$

We may define in the same spirit operators $\mathscr{U}_{a}$ and $\mathscr{D}_{a}$, which have the same effect on $f_{a}(u)$, the ath order fractional Fourier transform of $f(u)$ :

$$
\begin{aligned}
& \mathscr{U}_{a} f_{a}\left(u_{a}\right)=\left(\mathscr{U}_{a} f\right)_{a}\left(u_{a}\right)=u_{a} f_{a}\left(u_{a}\right), \\
& \mathscr{D}_{a} f_{a}\left(u_{a}\right)=\left(\mathscr{D}_{a} f\right)_{a}\left(u_{a}\right)=\frac{1}{i 2 \pi} \frac{d f_{a}\left(u_{a}\right)}{d u_{a}},
\end{aligned}
$$

where we have explicitly written $u_{a}$ to avoid confusion. The effect of these operators is to coordinate multiply and differentiate the fractional Fourier transform of $f(u)$, rather than $f(u)$ itself. Now, with these definitions, the fifth and sixth properties of Table 2 can be written as

$$
\begin{aligned}
& \mathscr{F}^{a}[\mathscr{U} f(u)]=\cos \phi\left(\mathscr{U}_{a} f\right)_{a}\left(u_{a}\right)-\sin \phi\left(\mathscr{D}_{a} f\right)_{a}\left(u_{a}\right), \\
& \mathscr{F}^{a}[\mathscr{D} f(u)]=\sin \phi\left(\mathscr{U}_{a} f\right)_{a}\left(u_{a}\right)+\cos \phi\left(\mathscr{D}_{a} f\right)_{a}\left(u_{a}\right) .
\end{aligned}
$$

$\left(\mathscr{U}_{a} f\right)_{a}\left(u_{a}\right)$ is simply the $u_{a}$ representation of $\mathscr{U}_{a} f$, which we also refer to as the representation of $\mathscr{u}_{a} f$ in the ath fractional Fourier domain. In the notation of quantum mechanics, $\left(\mathscr{U}_{a} f\right)_{a}\left(u_{a}\right)$ would have been written as $\left\langle u_{a} \mid \mathscr{U}_{a} f\right\rangle$. Similar comments apply to $\left(\mathscr{D}_{a} f\right)_{a}\left(u_{a}\right)$. The two preceding equations can be written in abstract operator form as

$$
\left[\begin{array}{l}
\mathscr{U} \\
\mathscr{D}
\end{array}\right]=\left[\begin{array}{rr}
\cos \phi & -\sin \phi \\
\sin \phi & \cos \phi
\end{array}\right]\left[\begin{array}{l}
\mathscr{U}_{a} \\
\mathscr{D}_{a}
\end{array}\right], \quad \phi=\frac{a \pi}{2} .
$$

We see that the coordinate multiplication and differentiation operators corresponding to order $a$ are related to those in the ordinary space (or time) domain by a simple rotation matrix.

The commutator $[\mathscr{U}, \mathscr{D}] \equiv \mathscr{U} \mathscr{D}-\mathscr{D} \mathscr{U}$ is well known to be equal to $i / 2 \pi$. By using Equation 44 we can easily derive the commutator [Aytür and Ozaktas, 1995; Ozaktas and Aytür, 1995]:

$$
\left[\mathscr{U}_{a}, \mathscr{U}_{a^{\prime}}\right]=\left(\frac{i}{2 \pi}\right) \sin \left(\phi^{\prime}-\phi\right)
$$

Knowing the commutator of two operators allows one to deduce an uncertainty relation between the two representations associated with those 
operators. In particular, the above commutation relation leads to [Aytuir and Ozaktas, 1995; Ozaktas and Aytür, 1995]

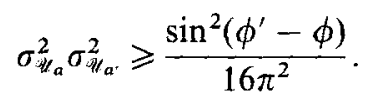

Here $\sigma_{\Psi_{a}}$ is the standard deviation of $\left|f_{a}\left(u_{a}\right)\right|^{2}$ and $\sigma_{\mathscr{H}_{a}}$ is the standard deviation of $\left|f_{a^{\prime}}\left(u_{a^{\prime}}\right)\right|^{2}$.

The translation and phase shift operators can also be expressed in operator notation. Let $\mathscr{T}(\xi)$ denote the operator which takes $f(u)$ to $f(u-\xi)$ and let $\mathscr{P}(\xi)$ denote the operator which takes $f(u)$ to $\exp (i 2 \pi \xi u) f(u)$, all in the ordinary space domain. We may also define $\mathscr{T}_{a}(\xi)$ and $\mathscr{P}_{a}(\xi)$ as the operators which have the same effect on the ath order fractional Fourier transforms: $\mathscr{T}_{a}(\xi)$ takes $f_{a}\left(u_{a}\right)$ to $f_{a}\left(u_{a}-\xi\right)$ and $\mathscr{P}_{a}(\xi)$ takes $f_{a}\left(u_{a}\right)$ to $\exp \left(i 2 \pi \xi u_{a}\right) f_{a}\left(u_{a}\right)$. Then the third and fourth properties of Table 2 can be expressed as [Aytür and Ozaktas, 1995; Ozaktas and Aytür, 1995]

$$
\begin{aligned}
\mathscr{F}^{a}[\mathscr{T}(\xi) f(u)] & =e^{i \pi \xi^{2} \sin \phi \cos \phi}\left[\mathscr{P}_{a}(-\xi \sin \phi) \mathscr{T}_{a}(\xi \cos \phi) f\right]_{a}\left(u_{a}\right) \\
& =e^{-i \pi \xi^{2} \sin \phi \cos \phi}\left[\mathscr{T}_{a}(\xi \cos \phi) \mathscr{P}_{a}(-\xi \sin \phi) f\right]_{a}\left(u_{a}\right), \\
\mathscr{F}^{a}[\mathscr{P}(\xi) f(u)] & =e^{-i \pi \xi^{2} \sin \phi \cos \phi}\left[\mathscr{P}_{a}(\xi \cos \phi) \mathscr{T}_{a}(\xi \sin \phi) f\right]_{a}\left(u_{a}\right) \\
& =e^{+i \pi \xi^{2} \sin \phi \cos \phi}\left[\mathscr{T}_{a}(\xi \sin \phi) \mathscr{P}_{a}(\xi \cos \phi) f\right]_{a}\left(u_{a}\right) .
\end{aligned}
$$

Here again the notation $[\mathscr{A} f]_{a}\left(u_{a}\right)$, where $\mathscr{A}$ is some operator, denotes the $u_{a}$ representation of $\mathscr{A} f$, which would be written as $\left.\left\langle u_{a}\right| \mathscr{A} f\right)$ in quantum mechanics. In operator form

$$
\begin{aligned}
\mathscr{T}(\xi) & =e^{i \pi \xi^{2} \sin \phi \cos \phi} \mathscr{P}_{a}(-\xi \sin \phi) \mathscr{T}_{a}(\xi \cos \phi) \\
& =e^{-i \pi \xi^{2} \sin \phi \cos \phi} \mathscr{T}_{a}(\xi \cos \phi) \mathscr{P}_{a}(-\xi \sin \phi), \\
\mathscr{P}(\xi) & =e^{-i \pi \xi^{2} \sin \phi \cos \phi} \mathscr{P}_{a}(\xi \cos \phi) \mathscr{T}_{a}(\xi \sin \phi) \\
& =e^{i \pi \xi^{2} \sin \phi \cos \phi} \mathscr{T}_{a}(\xi \sin \phi) \mathscr{P}_{a}(\xi \cos \phi) .
\end{aligned}
$$

We again see that the effect of translation is a combination of translation (by $\cos \phi$ ) and phase multiplication (by $\sin \phi$ ) of the fractional Fourier transform. A similar comment applies to the effect of phase multiplication. When $a=1$, these results reduce to the corresponding well-known properties of the ordinary Fourier transform.

The fractional Fourier transform does not have a convolution or multiplication property of comparable simplicity to that of the ordinary Fourier transform. 


\section{Vil. Relation to the Wigner Distribution}

The direct and simple relationship of the fractional Fourier transform to the Wigner distribution as well as to certain other phase-space distributions is perhaps its most important and elegant property [Mustard, 1989, 1996; Lohmann, 1993; Almeida, 1994; Mendlovic, Ozaktas, and Lohmann, 1994a; Ozaktas and others, 1994a].

Here we will define and briefly discuss some of the most important properties of the Wigner distribution. The Wigner distribution $W_{f}(u, \mu)$ of a function $f(u)$ is defined as

$$
W_{f}(u, \mu)=\int f\left(u+u^{\prime} / 2\right) f^{*}\left(u-u^{\prime} / 2\right) e^{-2 \pi \mu u^{\prime}} d u^{\prime} .
$$

$W_{f}(u, \mu)$ can also be expressed in terms of $F(\mu)$, or indeed as a function of any fractional transform of $f(u)$. Some of its most important properties are

$$
\begin{aligned}
|f(u)|^{2} & =\int W(u, \mu) d \mu, \\
|F(\mu)|^{2} & =\int W(u, \mu) d u, \\
\operatorname{En}[f(u)] & =\int W(u, \mu) d u d \mu .
\end{aligned}
$$

Roughly speaking, $W(u, \mu)$ can be interpreted as a function that indicates the distribution of the signal energy over space and frequency. The Wigner distribution of $F(u)$ (the Fourier transform of $f(u)$ ) is a ninety-degree rotated version of the Wigner distribution of $f(u)$. More on the Wigner distribution and other such distributions and representations may be found in Claasen and Mecklenbräuker [1980a,b,c, 1993], Hlawatsch and Boudreaux-Bartels [1992], and Cohen [1989, 1995].

Now, if $W_{f}(u, \mu)$ denotes the Wigner distribution of $f(u)$, then the Wigner distribution of the ath fractional Fourier transform of $f(u)$, denoted by $W_{f_{a}}(u, \mu)$, is given by

$$
W_{f_{a}}(u, \mu)=W_{f}(u \cos \phi-\mu \sin \phi, u \sin \phi+\mu \cos \phi) .
$$

so that the Wigner distribution of $W_{f_{a}}(u, \mu)$ is obtained from $W_{f}(u, \mu)$ by rotating it clockwise by an angle $\phi$. Let us define $\mathscr{R}_{\phi}$ to be the operator which rotates a function of $(u, \mu)$ by angle $\phi$ in the conventional counterclockwise direction. Then we can write

$$
W_{f_{\alpha}}(u, \mu)=\mathscr{R}_{-\phi} W_{f}(u, \mu) .
$$


This elegant and fundamental property underlies an important number of the applications of the fractional Fourier transform. In fact, some authors have defined the transform as that operation which corresponds to rotation of the Wigner distribution of a function [Lohmann, 1993].

Equation 56 can be derived directly from Equation 6 and the definition of the Wigner distribution given by Equation 52 [Ozaktas and others, 1994a]. The derivation is somewhat lengthy but straightforward. A similar derivation is given by Mustard [1989, 1996] and by Almeida [1994]. Lohmann [1993] shows the reverse, starting from the rotation property and arriving at Equation 6.

An at least equally important form of this result follows easily [Mustard, 1989, 1996; Lohmann and Soffer, 1994; Ozaktas and others, 1994a]. Let us recall Equations 53 and 54, which state that the integral projection of $W_{f}(u, \mu)$ onto the $u$ axis is the magnitude square of the $u$-domain representation of the signal and that the integral projection of $W_{f}(u, \mu)$ onto the $\mu$ axis is the magnitude square of the $\mu$-domain representation of the signal. Now, let us rewrite the first of these equations for $f_{a}(u)$, the ath order fractional Fourier transform of $f(u)$ :

$$
\int W_{f_{a}}(u, \mu) d \mu=\left|f_{a}(u)\right|^{2} .
$$

Since $W_{f_{a}}(u, \mu)$ is simply $W_{f}(u, \mu)$ clock wise rotated by angle $\phi$, the integral projection of $W_{f_{a}}(u, \mu)$ onto the $u$ axis is identical to the integral projection of $W_{f}(u, \mu)$ onto an axis making angle $\phi$ with the $u$ axis. This new axis making angle $\phi=a \pi / 2$ with the $u$ axis is referred to as the $u_{a}$ axis. Let $\mathscr{R} \mathscr{A} \mathscr{D}_{\phi}$ denote the Radon transform operator, which maps a two-dimensional function of $(u, \mu)$ to its integral projection onto an axis making angle $\phi$ with the $u$ axis [Bracewell, 1995]. Thus the above can be written as

$$
\mathscr{R} \mathscr{A}_{\mathscr{D}_{\phi}} W_{f}(u, \mu)=\left|f_{a}(u)\right|^{2} .
$$

In conclusion, the integral projection of the Wigner distribution of a function onto the $u_{a}$ axis is equal to the magnitude square of the ath order fractional Fourier transform of the function (Fig. 4). Equations 53 and 54 are special cases with $a=0$ and $a=1$. Wood and Barry discussed what they referred to as the "Radon-Wigner transform" without realizing its relation to the fractional Fourier transform [Wood and Barry, 1994a,b]. The above discussion demonstrates that the Radon-Wigner transform is simply the magnitude squared of the fractional Fourier transform.

The results of this section, and in particular Equation 59, continue to hold when $W_{f}(u, \mu)$ is the Wigner distribution of a random process since the expectation value operation can move inside the Radon transform and 


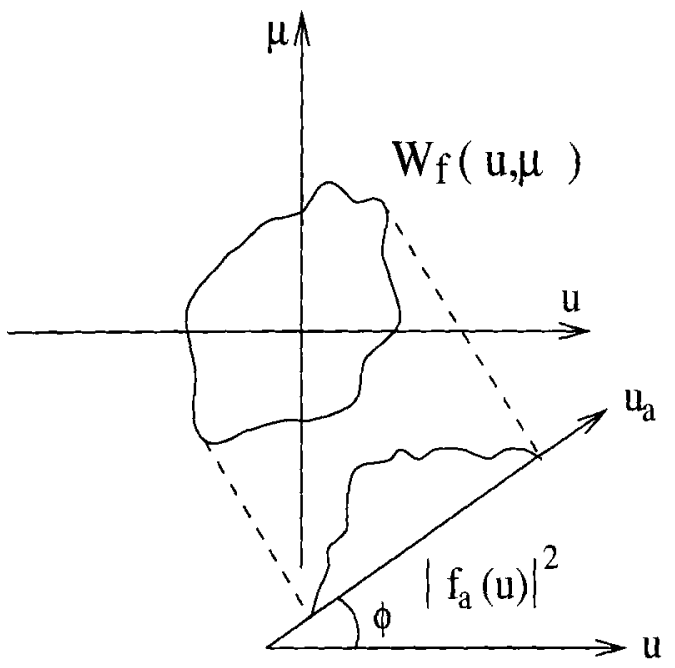

FiguRE 4. Oblique integral projections of the Wigner distribution.

rotation operators:

$$
\begin{aligned}
& \overline{W_{f_{a}}(u, \mu)}=\mathscr{R}_{-\phi} \overline{W_{f}(u, \mu)}, \\
& \mathscr{R} \mathscr{A} \mathscr{D}_{\phi} \overline{W_{f}(u, \mu)}=\overline{\left|f_{a}(u)\right|^{2}} .
\end{aligned}
$$

Here the overbars denote ensemble averages.

The Wigner distribution is not the only time-frequency representation satisfying the rotation property (Equation 57 ). The ambiguity function also satisfies this property because the ambiguity function is the two-dimensional Fourier transform of the Wigner distribution, and the two-dimensional Fourier transform of the rotated version of a function is the rotated version of the two-dimensional Fourier transform of the original function [Ozaktas and others, 1994a; Almeida, 1994]. Almeida [1994] showed that the rotation property also holds for the spectrogram. It has been further shown that the rotation property generalizes to certain other time-frequency distributions belonging to the so-called Cohen class, whose members can be obtained from the Wigner distribution by convolving it with a kernel characterizing that distribution. The distributions for which the rotation property holds are those which have a rotationally symmetric kernel [Ozaktas, Erkaya, and Kutay, 1996a].

Thus, fractional Fourier transformation corresponds to rotation of many phase-space representations. This not only confirms the important role this 
transform plays in the study of such representations but also supports the notion of referring to the axis making angle $\phi=a \pi / 2$ with the $u$ axis as the ath fractional Fourier domain. Despite this generalization, the only distribution which satisfies a relation of the form of Equation 59 is the Wigner distribution [Mustard, 1989]. Cohen [1989] argues that there is nothing special about the Wigner distribution among other members of the Cohen class, since all members of this class, including the Wigner distribution, are derivable from each other through convolution relations. However, the fact that only the Wigner distribution satisfies Equation 59 has led Mustard to suggest that the Wigner distribution is a specially distinguished member of the Cohen class [Mustard, 1989, 1996].

As an instructive application of the Wigner rotation property, we discuss the fractional Fourier transforms of chirp functions. The transform of the chirp function $\exp \left[i \pi\left(\chi u^{2}+2 \xi u\right)\right]$ was given in Table 1 as a rather complicated expression. Phase-space offers a much more transparent picture. The Wigner distribution of the chirp function $\exp \left[i \pi\left(\chi u^{2}+2 \xi u\right)\right]$ is $W_{f}(u, \mu)=$ $\delta(\mu-\chi u-\xi)$, which is simply a line delta in phase space along the line $\mu=$ $\chi u+\xi$ making angle orctan $(\chi)$ with the $u$ axis (Fig. 5). The Wigner distribution of $\exp (i 2 \pi \xi u)$ is $W_{f}(u, \mu)=\delta(\mu-\xi)$ and is seen to be a special case in which the line delta is horizontal. Similarly, the Wigner distribution of $\delta(u-\xi)$ is $W_{f}(u, \mu)=\delta(u-\xi)$ and is also seen to be a special case in

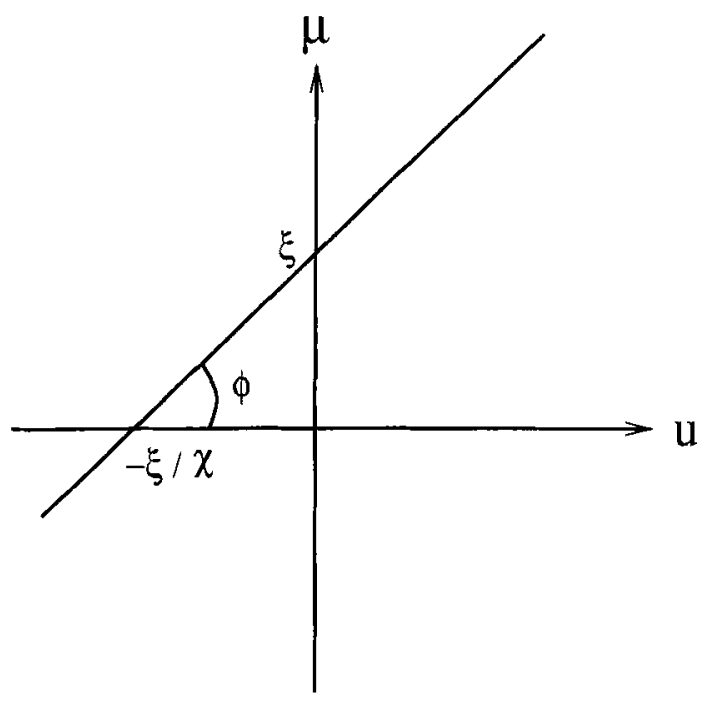

FIGURE 5. Wigner distribution of a chirp function. 
which the line delta is vertical. (That the chirp indeed behaves like a delta function as $\chi, \xi \rightarrow \infty$ can be shown by using Equation 17.) Thus, since harmonic functions and delta functions can be considered degenerate or limiting cases of chirp functions, it is possible to make the general statement that the fractional Fourier transform of a chirp function is always another chirp function. In phase space, we observe this as the rotation of line deltas into other line deltas.

\section{Fractional Fourier Domains}

Equations 57 and 59 immediately lead to the interpretation of oblique axes in phase space as fractional Fourier domains. Just as the projection of the Wigner distribution onto the space domain gives the magnitude square of the space-domain representation of the signal, and the projection of the Wigner distribution onto the frequency domain gives the magnitude square of the frequency-domain representation of the signal (Equations 53 and 54), the projection onto the axis making angle $\phi=a \pi / 2$ with the $u$ axis gives the magnitude square of the ath fractional Fourier-domain representation of the signal (Equation 59). When we need to be explicit we will use the variable $u_{a}$ as the coordinate variable in the $a$ th domain, so that the representation of the signal $f$ in the ath order fractional Fourier domain will be written as $f_{a}\left(u_{a}\right)$. We immediately recognize that the 0th and 1st domains are the ordinary space and frequency domains and that the 2nd and 3rd domains correspond to the negated space and frequency domains $\left(u_{0}=u, u_{1}=\mu\right.$, $u_{2}=-u, u_{3}=-\mu$ ). The representation of the signal in the $a^{\prime}$ th domain is related to its representation in the ath domain through an $\left(a^{\prime}-a\right)$ th order fractional Fourier transformation:

$$
f_{a^{\prime}}\left(u_{a^{\prime}}\right)=\int K_{a^{\prime}-a}\left(u_{a^{\prime}}, u_{a}\right) f_{a}\left(u_{a}\right) d u_{a}
$$

When $\left(a^{\prime}-a\right)$ is an integer, this corresponds to a forward or inverse Fourier integral.

The notion of fractional Fourier domains as oblique axes in spacefrequency is also confirmed by the operational formula presented earlier. We had seen that a translation of $u_{0}$ corresponded to $\cos \phi$ much of the shift and a $\sin \phi$-much phase shift in the ath domain. Likewise, we had seen that multiplication by $u$ corresponded to $\cos \phi$ much of the multiplication and a $\sin \phi$-much differentiation. 


\section{Differential Equations}

Here we will see that the fractional Fourier transform $f_{a}(u)$ of a function $f_{0}(u)$ is the solution of a differential equation, where $f_{0}(u)$ may be interpreted as the initial condition of the equation. This is the quantummechanical harmonic oscillator differential equation and also the equation governing optical propagation in quadratic graded-index media (in the former case the order parameter $a$ corresponds to time and in the latter case it corresponds to the coordinate along the direction of propagation) [Agarwal and Simon, 1994; Ozaktas and Mendlovic, 1995]. In fact, in some sources the solution is written in the form of an integral transform whose kernel is sometimes referred to as the harmonic oscillator Green's function, without the authors knowing that this is the fractional Fourier transform. (To be precise, we must note that the differential equations governing these physical phenomena differ slightly from the equation we discuss, but this small difference is inconsequential.)

The differential equation is

$$
i \frac{2}{\pi} \frac{\partial f_{a}(u)}{\partial a}=\left[-\frac{1}{4 \pi} \frac{\partial^{2}}{\partial u^{2}}+\pi u^{2}-\frac{1}{2}\right] f_{a}(u),
$$

with the initial condition $f_{0}(u)=f(u)$. The solution $f_{a}(u)$ of the equation is the $a$ th order fractional Fourier transform of $f(u)$ as can be shown by direct substitution of Equation 6.

Alternatively, and more instructively, we may take an eigenvalue equation approach. Substituting the form $f_{a}(u)=\exp (-i \beta a) f_{0}(u)$ in Equation 63, we obtain

$$
\frac{d^{2} f_{0}(u)}{d u^{2}}+4 \pi^{2}\left(\frac{1}{2 \pi}+\frac{2 \beta}{\pi^{2}}-u^{2}\right) f_{0}(u)=0 .
$$

Comparing this equation with the standard equation

$$
\frac{d^{2} f(u)}{d u^{2}}+4 \pi^{2}\left(\frac{2 n+1}{2 \pi}-u^{2}\right) f(u)=0,
$$

whose solutions are well known as the Hermite-Gaussian functions $\psi_{n}(u)$, we conclude that the $n$th Hermite-Gaussian function is a solution of Equation 64 when $\beta=\beta_{n}=n \pi / 2$. It is now possible to write down arbitrary solutions of the equation as a linear superposition of these eigenfunctions (modes). If the initial condition $f_{0}(u)$ is $\psi_{n}(u)$, the solution $f_{a}(u)$ is $\exp (-\operatorname{ian} \pi / 2) \psi_{n}(u)$; this is what it means to be an eigenfunction. Given an arbitrary initial condition $f_{0}(u)$, we can expand it in terms of the Hermite- 
Gaussian functions $\psi_{n}(u)$ as

$$
\begin{aligned}
f_{0}(u) & =\sum_{n=0}^{\infty} C_{n} \psi_{n}(u), \\
C_{n} & =\int \psi_{n}(u) f_{0}(u) d u .
\end{aligned}
$$

Since Equation 63 is linear, the solution corresponding to this initial condition is readily obtained as

$$
f_{a}(u)=\sum_{n=0}^{\infty} C_{n} e^{-i a n \pi / 2} \psi_{n}(u)
$$

from which one can obtain

$$
\begin{gathered}
f_{a}(u)=\int K_{a}\left(u, u^{\prime}\right) f_{0}\left(u^{\prime}\right) d u^{\prime}, \\
K_{a}\left(u, u^{\prime}\right)=\sum_{n=0}^{\infty} e^{-i a n \pi / 2} \psi_{n}(u) \psi_{n}\left(u^{\prime}\right),
\end{gathered}
$$

exactly as in Equation 32.

The differential equation in question can also be written in terms of the operators $\mathscr{U}$ and $\mathscr{D}$ as follows:

$$
\pi\left(\mathscr{U}^{2}+\mathscr{D}^{2}\right) f_{a}(u)-\frac{1}{2} f_{a}(u)=i \frac{\partial f_{a}(u)}{\partial(\pi a / 2)},
$$

or

$$
\mathscr{H} f_{a}(u)=i \frac{\partial f_{a}(u)}{\partial(\pi a / 2)} .
$$

Readers with a background in quantum mechanics will readily recognize the Hamiltonian $\mathscr{H}=\pi\left(\mathscr{U}^{2}+\mathscr{D}^{2}\right)-1 / 2$ which characterizes the harmonic oscillator. This Hamiltonian is domain-invariant, which means that $\pi\left(\mathscr{U}_{a}^{2}+\mathscr{D}_{a}^{2}\right)$ is the same operator regardless of the value of $a$, as can be readily shown by using Equation 44 . This rotational invariance of the Hamiltonian ties in with the Wigner rotation property. (The extra $-1 / 2$ represents the inconsequential discrepancy mentioned earlier.)

More on the relationship of the fractional Fourier transform to differential equations and their solutions is found in Namias [1980] and McBride and Kerr [1987]. 


\section{HyperdifFerential Form}

The hyperdifferential form of the fractional Fourier transform operator is given by [Namias, 1980; Mustard, 1987a].

$$
\begin{aligned}
\mathscr{F}^{a} & =e^{-i(a \pi / 2) *}, \\
\mathscr{H} & =\pi\left(\mathscr{D}^{2}+\mathscr{U}^{2}\right)-\frac{1}{2} .
\end{aligned}
$$

Applied to a function $f(u)$ we may write

$$
\mathscr{F}^{a} f(u)=\exp \left[-i\left(\frac{a \pi}{2}\right)\left(-\frac{1}{4 \pi} \frac{d^{2}}{d u^{2}}+\pi u^{2}-\frac{1}{2}\right)\right] f(u) .
$$

This hyperdifferential representation may be considered the formal solution of the differential equation written in the form of Equation 71. The operator $\mathscr{F}^{a}$ given in Equation 72 generates $f_{a}(u)$ for all values of $a$ from $f_{0}(u)=f(u)$. The index additivity property and the special case $a=0$ immediately follow from the exponential form $\exp (-i \phi \mathscr{H})$.

\section{Xi. Digital Simulation of the Transform}

Here we briefly discuss how the fractional Fourier transform may be computed on a digital computer, referring the reader to Ozaktas and others [1996b] for further details.

The defining equation (Equation 6) can be put in the form

$$
f_{a}(u)=A_{\phi} e^{i \pi \cot \phi u^{2}} \int e^{-i 2 \pi \csc \phi u u^{\prime}}\left[e^{i \pi \cot \phi u^{\prime 2}} f\left(u^{\prime}\right)\right] d u^{\prime} .
$$

We assume that the representations $f_{a}\left(u_{a}\right)$ of the signal $f$ in all fractional Fourier domains are approximately confined to the interval $[-\Delta u / 2, \Delta u / 2]$ (that is, a sufficiently large percentage of the signal energy is confined to these intervals). This assumption is equivalent to assuming that the Wigner distribution of $f(u)$ is approximately confined within a circle of diameter $\Delta u$ (by virtue of Equation 59). Again, this means that a sufficiently large percentage of the energy of the signal is contained in that circle. We can ensure that this assumption is valid for any signal by choosing $\Delta u$ sufficiently large. Under this assumption and initially limiting the order $a$ to the interval $0.5 \leqslant|a| \leqslant 1.5$, the modulated function $e^{i \pi \alpha u^{\prime 2}} f\left(u^{\prime}\right)$ may be assumed to be approximately band-limited to $\pm \Delta u$ in the frequency domain. Thus 
$e^{i \pi \alpha u^{\prime 2}} f\left(u^{\prime}\right)$ can be represented by Shannon's interpolation formula

$$
e^{i \pi \alpha u^{\prime 2}} f\left(u^{\prime}\right)=\sum_{n=-N}^{N-1} e^{i \pi \cot \phi(n / 2 \Delta u)^{2}} f\left(\frac{n}{2 \Delta u}\right) \operatorname{sinc}\left[2 \Delta u\left(u^{\prime}-\frac{n}{2 \Delta u}\right)\right],
$$

where $N=(\Delta u)^{2}$. The summation goes from $-N$ to $N-1$ since $f\left(u^{\prime}\right)$ is assumed to be zero outside $[-\Delta u / 2, \Delta u / 2]$. By using Equation 75 and Equation 74 and changing the order of integration and summation, we obtain

$$
\begin{aligned}
f_{a}(u)= & A_{\phi} e^{i \pi \cot \phi u^{2}} \sum_{n=-N}^{N-1} e^{i \pi \cot \phi(n / 2 \Delta u)^{2}} f\left(\frac{n}{2 \Delta u}\right) \int e^{-i 2 \pi \csc \phi u u^{\prime}} \\
& \times \operatorname{sinc}\left[2 \Delta u\left(u^{\prime}-\frac{n}{2 \Delta u}\right)\right] d u^{\prime} .
\end{aligned}
$$

By recognizing the integral to be equal to $(1 / 2 \Delta n) e^{-i 2 \pi \csc \phi u(n / 2 \Delta u)}$ $\operatorname{rect}(\csc (\phi) u / 2 \Delta u)$, we can write

$$
f_{a}(u)=\frac{A_{\phi}}{2 \Delta u} \sum_{n=-N}^{N-1} e^{i \pi \cot \phi u^{2}} e^{-i 2 \pi \csc \phi u(n / 2 \Delta u)} e^{i \pi \cot \phi(n / 2 \Delta u)^{2}} f\left(\frac{u}{2 \Delta u}\right),
$$

since $\operatorname{rect}(\csc (\phi) / 2 \Delta u)=1$ in the interval $|u| \leqslant \Delta u / 2$. Then, the samples of $f_{a}(u)$ are given by

$f_{a}\left(\frac{m}{2 \Delta u}\right)=\frac{A_{\phi}}{2 \Delta u} \sum_{n=-N}^{N-1} e^{i \pi\left(\cot \phi(m / 2 \Delta u)^{2}-2 \csc \phi m n /(2 \Delta u)^{2}+\cot \phi(n / 2 \Delta u)^{2}\right)} f\left(\frac{n}{2 \Delta u}\right)$,

which is a finite summation allowing us to obtain the samples of the fractional transform $f_{a}(u)$ in terms of the samples of the original function $f(u)$. Direct computation of Equation 78 would require $O\left(N^{2}\right)$ operations. A fast $(O(N \log N))$ algorithm can be obtained by putting Equation 78 into the following form:

$$
\begin{aligned}
f_{a}\left(\frac{m}{2 \Delta u}\right)= & \frac{A_{\phi}}{2 \Delta u} e^{i \pi(\cot \phi-\csc \phi)(m / 2 \Delta u)^{2}} \sum_{n=-N}^{N-1} e^{i \pi \csc \phi((m-n) / 2 \Delta u)^{2}} \\
& \times e^{i \pi(\cot \phi-\csc \phi)(n / 2 \Delta u)^{2}} f\left(\frac{n}{2 \Delta u}\right) .
\end{aligned}
$$

We now recognize that the summation is the convolution of $e^{i \pi \csc \phi(n / 2 \Delta u)^{2}}$ and the chirp-modulated function $f(\cdot)$. The convolution can be computed in $O(N \log N)$ time by using the fast Fourier transform (FFT). The output samples are then obtained by a final chirp modulation. Hence the overall complexity is $O(N \log N)$.

We had limited ourselves to $0.5 \leqslant|a| \leqslant 1.5$ in deriving the above algorithm. Using the index additivity property of the fractional Fourier trans- 
form we can extend this range to all values of $a$ easily. For instance, for the range $0 \leqslant a \leqslant 0.5$, we can write

$$
\mathscr{\mathscr { F }}^{a}=\mathscr{F}^{a-1+1}=\mathscr{\mathscr { F }}^{a-1} \mathscr{F}^{-1} .
$$

Since $0.5 \leqslant|a-1| \leqslant 1$, we can use the above algorithm in conjunction with the ordinary Fourier transform to compute $f_{a}(u)$. The overall complexity remains at $O(N \log N)$.

\section{Xil. Applications to Wave and Beam Propagation}

A considerable number of papers have been written on the application of the fractional Fourier transform to wave and beam propagation problems, mostly in an optical context. Our presentation will also be phrased in the notation and terminology of optics. Nevertheless, the reader should have no difficulty translating the results to other propagation, diffaction, and scattering phenomena which are mathematically equivalent or similar.

Whenever we can express the result of an optical problem (such as Fraunhofer diffraction) in terms of a Fourier transform, we tend to think of this as a simple and elegant result. This is justified by the fact that the Fourier transform has many simple and useful properties which make it attractive to work with. The Fourier transform and image occur at certain privileged planes in an optical system. Often all our intuition about what happens in between these planes is that the amplitude distribution is given by a complicated integral. We will see below that the distribution of light at intermediate planes can be expressed in terms of the fractional Fourier transform (which also has several useful properties and operational formulas). Thus the fractional Fourier transform completes in a very natural way the study of optical systems often called "Fourier optics."

Fourier optical systems can be analyzed using geometrical optics, Fresnel integrals (spherical wave expansions), plane wave expansions. HermiteGaussian beam expansions, and, as we will discuss, fractional Fourier transforms. The several approaches prove useful in different situations and provide different viewpoints which complement each other. The fractional Fourier transform approach is appealing in that it describes the continuous evolution of the wave as it propagates through the system.

\section{A. Introduction}

Optical systems involving an arbitrary sequence of thin lenses separated by arbitrary sections of free-space (under the Fresnel approximation) belong to the class of quadratic-phase systems. Mathematically, quadratic-phase sys- 
tems are equivalent to linear canonical transforms [Wolf, 1979]. Systems contain arbitrary sections of quadratic graded-index media also belong to this class. The class of Fourier optical systems (or first order optical systems) consist of arbitrary thin filters sandwiched betwen arbitrary quadratic-phase systems. Members of the class of quadratic-phase systems are characterized by linear transformations of the form [Bastiaans, 1978, 1979a, 1979b, 1989, 1991; Nazarathy and Shamir, 1982; Ozaktas and Mendlovic, 1995]

$$
\begin{aligned}
f_{\text {out }}(u) & =\int h\left(u, u^{\prime}\right) f_{\text {in }}\left(u^{\prime}\right) d u^{\prime} \\
h\left(u, u^{\prime}\right) & =K^{\prime} \exp \left[i \pi\left(\alpha u^{2}-2 \beta u u^{\prime}+\gamma u^{\prime 2}\right)\right],
\end{aligned}
$$

where $K^{\prime}$ is a complex constant and $\alpha, \beta$, and $\gamma$ are real constants. Comparing this equation with Equation 6, we see that fractional Fourier transforms are a special case of quadratic-phase systems.

Until this point, all variables have been considered to be dimensionless and were denoted by $u$, $\mu$, etc., and all functions and kernels took dimensionless arguments and were denoted by $f(u), g(u), h\left(u, u^{\prime}\right)$, etc. In optical applications we will often employ variables with the dimensions of length or inverse length, which we will denote by $x$, $p$, etc. Functions and kernels taking such arguments will be distinguished as $\hat{f}(u), \hat{g}(u), \hat{h}\left(u, u^{\prime}\right)$, etc. With these conventions, Equation 81 can be rewritten as

$$
\begin{aligned}
\hat{f}_{\text {out }}(x) & =\int \hat{h}\left(x, x^{\prime}\right) \hat{f}_{\text {in }}\left(x^{\prime}\right) d x^{\prime}, \\
\hat{h}\left(x, x^{\prime}\right) & =K \exp \left[\frac{i \pi}{s^{2}}\left(\alpha x^{2}-2 \beta x x^{\prime}+\gamma x^{\prime 2}\right)\right],
\end{aligned}
$$

where $K=K^{\prime} / s, s$ is a constant with the dimension of length, and $\hat{f}_{\text {our }}(x) \equiv$ $f_{\text {out }}(x / s)$, etc. The choice of $s$ essentially corresponds to the choice of units. We will assume $s$ is specified once and for all throughout our analysis.

The kernels associated with a thin lens with focal length $f$ and free-space propagation over a distance $d$ are given respectively by [Saleh and Teich, 1991]

$$
\begin{aligned}
\hat{h}_{\text {lens }}\left(x, x^{\prime}\right) & =K_{\text {lens }} \delta\left(x-x^{\prime}\right) \exp \left(-i \pi x^{2} / \lambda f\right), \\
\hat{h}_{\text {space }}\left(x, x^{\prime}\right) & =K_{\text {space }} \exp \left[i \pi\left(x-x^{\prime}\right)^{2} / \lambda d \mid .\right.
\end{aligned}
$$

$\lambda$ is the wavelength of light in free space and $K_{\text {lens }}$ and $K_{\text {space }}$ are constants. We continue to work with one-dimensional notation for simplicity, although 
most optical systems are two-dimensional. These kernels are special cases of the kernel given in Equation 81. It is possible to prove that any arbitrary concatenation of kernels of this form will result in a kernel of the form given in Equation 82.

Apart from the constant factor $K$, which has no effect on the resulting spatial distribution, a member of the class of quadratic-phase systems is completely specified by the three parameters $\alpha, \beta$, and $\gamma$ (Equation 81). Alternatively, such a system can also be completely specified by the transformation matrix [Bastiaans, 1989; Nazarathy and Shamir, 1982; Ozaktas and Mendlovic, 1995]

$$
\mathbf{T} \equiv\left[\begin{array}{ll}
A & B \\
C & D
\end{array}\right] \equiv\left[\begin{array}{cc}
\gamma / \beta & s^{2} / \beta \\
-\beta / s^{2}+\alpha \gamma / s^{2} \beta & \alpha / \beta
\end{array}\right]
$$

with $A D-B C=1$. Here again $s$ is the scale factor relating our dimensional and dimensionless variables. If several systems, each characterized by such a matrix, are cascaded, the matrix characterizing the overall system can be found by multiplying the matrices of the several systems. The matrix defined above also corresponds to the well-known ray matrix employed in ray optical analysis. At a certain plane perpendicular to the optical axis, a ray can be characterized by its distance from the optical axis $z$ and its paraxial angle of inclination $\theta$. We will define the ray vector as $\left[\begin{array}{ll}x & p\end{array}\right]^{\mathrm{T}}$ where $p \equiv \theta / \lambda$. Then, the ray vector at the output is related to the ray vector at the input by

$$
\left[\begin{array}{l}
x_{\text {out }} \\
p_{\text {out }}
\end{array}\right]=\left[\begin{array}{ll}
A & B \\
C & D
\end{array}\right]\left[\begin{array}{l}
x_{\text {in }} \\
p_{\text {in }}
\end{array}\right] .
$$

The matrices corresponding to a thin lens and a section of free-space are given respectively by

$$
\left[\begin{array}{ll}
A & B \\
C & D
\end{array}\right]=\left[\begin{array}{cc}
1 & 0 \\
-1 / \lambda f & 1
\end{array}\right]
$$

and

$$
\left[\begin{array}{ll}
A & B \\
C & D
\end{array}\right]=\left[\begin{array}{cc}
1 & \lambda d \\
0 & 1
\end{array}\right] .
$$

Quadratic graded-index media exhibit a parabolic refractive index profile $n(x)$ about the optical axis, characterized by the two parameters $n_{0}$ and $\eta$ as follows:

$$
n^{2}(x)=n_{0}^{2}\left(1-(x / \eta)^{2}\right)
$$


The matrix corresponding to quadratic graded-index media is given by

$$
\left[\begin{array}{ll}
A & B \\
C & D
\end{array}\right]=\left[\begin{array}{cc}
\cos \left[\left(d / d_{0}\right) \pi / 2\right. & \left(\eta \lambda / n_{0}\right) \sin \left[\left(d / d_{0}\right) \pi / 2\right] \\
-\left(n \lambda / n_{0}\right)^{-1} \sin \left[\left(d / d_{0}\right) \pi / 2\right] & \cos \left[\left(d / d_{0}\right) \pi / 2\right]
\end{array}\right],
$$

where $d$ is the length of the medium and $d_{0}=\eta \pi / 2$. This matrix can be derived by a simple application of the ray equation [Saleh and Teich, 1991].

The space spanned by the coordinates $x$ and $p$ also constitutes a phase-space which directly corresponds to the space-frequency plane on which the Wigner distribution was defined. A particular ray characterized by its phase-space vector $\left[\begin{array}{ll}x & p\end{array}\right]^{\mathrm{T}}$ will be mapped to another according to its $A B C D$ matrix given above. If we consider a bundle of rays constituting a region in this phase-space, this region will likewise be transformed according to the same matrix. For instance, let us consider the rectangular bundle consisting of rays whose intercepts lie between $-x_{0}$ and $x_{0}$ and whose inclinations lie between $-p_{0}=-\theta_{0} \lambda$ and $p_{0}=\theta_{0} \lambda$ (Fig. 6a). If this bundle passes through a lens, it will be transformed according to Equation 87 into the bundle shown in Fig. 6b. If this bundle passes through a section of free-space, it will be transformed according to Equation 88 into the bundle shown in Fig. 6c. More generally, for arbitrary $A B C D$ it will be transformed into a bundle of the general form shown in Fig. 6d.

This mapping of phase-space regions can also be posed in terms of the Wigner distribution. It is known that if $\hat{\jmath}_{\text {out }}$ is the linear canonical transform of $\vec{f}_{\text {in }}$ with parameters $A B C D$, then the Wigner distributon of $\vec{f}_{\text {out }}$ is related to that of $\hat{f}_{\text {in }}$ by the relation [Bastiaans, 1979b]

$$
\hat{W}_{\text {out }}\left(x_{\text {out }}, p_{\text {out }}\right)=\hat{W}_{\text {in }}\left(x_{\text {in }}, p_{\text {in }}\right) \text {. }
$$

where $x_{\text {out }}, p_{\text {out }}$ and $x_{\text {in }}, p_{\text {in }}$ are related according to Equation 86 . This is simply a generalization of the Wigner rotation property discussed in Section VII. In particular, since we know from this property that fractional Fourier transformation corresponds to rotation in phase-space, it follows that the $A B C D$ matrix for fractional Fourier transforms should be the rotation matrix.

\section{B. Quadratic-Phase Systems as Fractional Fourier Transforms}

As is evident by comparing Equations 6 and 81, the one-parameter class of fractional Fourier transforms is a subclass of the class of three-parameter quadratic-phase systems. If we allow an additional magnification parameter $M$ and a phase curvature parameter $1 / R$, the family of fractional Fourier transforms will now also have three parameters and can be put in one-toone correspondence with the family of quadratic-phase systems. The kernel 


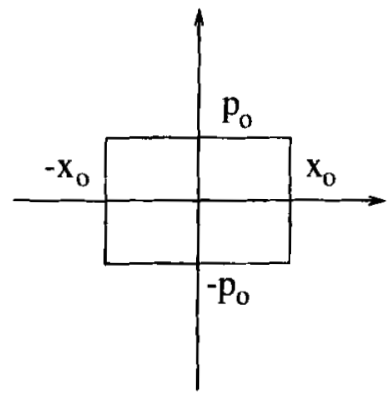

(a)

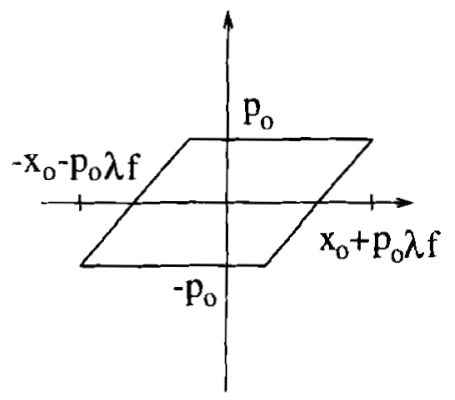

(c)

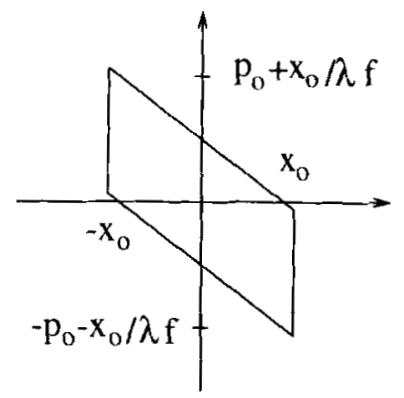

(b)

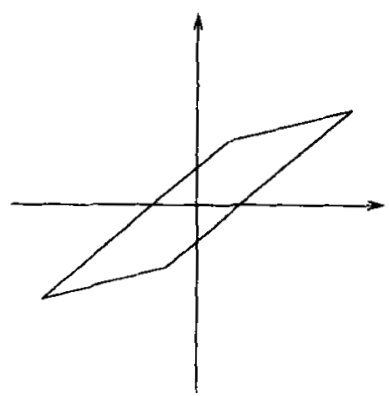

(d)

Figure 6. Effect of quadratic-phase systems in phase-space.

of this three-parameter transform may be written as

$$
\begin{aligned}
\hat{f}_{\text {out }}(x) & =\int \hat{h}\left(x, x^{\prime}\right) \hat{f}_{\text {in }}\left(x^{\prime}\right) d x^{\prime} \\
\hat{h}\left(x, x^{\prime}\right) & =K_{\mathrm{ff}} \exp \left(i \pi x^{2} / \lambda R\right) \exp \left[\frac{i \pi}{s^{2}}\left(\frac{x^{2}}{M^{2}} \cot \phi-2 \frac{x x^{\prime}}{M} \csc \phi+x^{\prime 2} \cot \phi\right)\right],
\end{aligned}
$$

which is in the form of a quadratic-phase system. (The pure mathematical form given by Equation 6 is recovered by setting $x / s=u, M=1, R=\infty$.) This kernel maps a function $f(x / s)$ into $K^{\prime} \exp \left(i \pi x^{2} / \lambda R\right) f_{a}(x / s M)$, where $f_{a}(u)$ is the $a$ th order fractional Fourier transform of $f(u)$. Here $\phi=a \pi / 2$ as before, $M>0$ is referred to as the magnification associated with the transform, and $R$ is the radius of the spherical surface on which the perfect 
fractional Fourier transform is observed. When $R=\infty$, the quadratic-phase term disappears and the perfect fractional Fourier transform is observed on a planar surface.

The above family of kernels is in one-to-one correspondence with the family of kernels given in Equation 81. The parameters $\alpha, \beta$, and $\gamma$ are recognized to be related to the parameters $\phi, M$, and $R$ through the relations

$$
\begin{aligned}
& \alpha=\cot \phi / M^{2}+s^{2} / \lambda R, \\
& \beta=\csc \phi / M, \\
& \gamma=\cot \phi .
\end{aligned}
$$

Alternatively, the $A B C D$ parameters are related to $\phi, M$, and $R$ through the relations

$\left[\begin{array}{ll}A & B \\ C & D\end{array}\right]==\left[\begin{array}{cc}M \cos \phi & s^{2} M \sin \phi \\ -\sin \phi / s^{2} M+M \cos \phi / \lambda R & \cos \phi / M+s^{2} M \sin \phi / \lambda R\end{array}\right]$,

which can be inverted to yield

$$
\begin{aligned}
\tan \phi & =\frac{1}{s^{2}} \frac{B}{A}, \\
M & =\sqrt{A^{2}+\left(B / s^{2}\right)^{2}}, \\
\frac{1}{\lambda R} & =\frac{1}{s^{4}} \frac{B / A}{A^{2}+\left(B / s^{2}\right)^{2}}+\frac{C}{A} .
\end{aligned}
$$

The above result essentially means that any quadratic-phase system can be interpreted as a magnified fractional Fourier transform, perhaps with a residual phase curvature. Since a relatively large class of optical systems can be modeled as quadratic-phase systems, these systems can also be interpreted as fractional Fourier transforms [Ozaktas and Mendlovic, 1996]. We will first consider two elementary examples --propagation in quadratic graded-index media and diffraction in free-space - and then treat the more general case of arbitrary composition of thin lenses and sections of freespace.

\section{Propagation in Quadratic Graded-Index Media}

Quadratic graded-index media have a natural and direct relationship with the fractional Fourier transform. Light is simply fractional Fourier transformed as it propagates through such media. The refractive index distribu- 
tion for such media was already given in equation 89 .

The $A B C D$ matrix for graded-index media was given as Equation 90 . Comparing this with Equation 96, we immediately conclude that propagation through a section of graded-index media results in a fractional Fourier transform of order $a=2 d / \pi \eta \equiv d / d_{0}$, provided the scale parameter $s$ is chosen such that $s^{2}=\lambda \eta / n_{0}$. Agreeing on this choice of $s$, there is no magnification $(M=1)$ and no residual phase curvature $(R=\infty)$. Recalling the comment at the end of Subsection A, we conclude that as light propagates through quadratic graded-index media, its Wigner distribution rotates. Quadratic graded-index media realize fractional Fourier transforms in their purest and simplest form. If the distribution of light at the input plane is given by $f(x / s)$, then the distribution of light at the output plane is proportional to $f_{a}(x / s)$, where the transform order $a$ increases linearly with distance of propagation.

The same result can be arrived at by starting from the Helmholtz equation for quadratic graded-index media, finding its modes (which are the Hermite-Gaussian functions), and constructing arbitrary solutions as linear superpositions of these modes. This approach may be found in Mendlovic and Ozaktas [1993] and Ozaktas and Mendlovic [1993a,b].

\section{Fresnel Diffraction}

Although quadratic graded-index media are perfectly matched to the fractional Fourier transform, it is of interest to discuss the more basic problem of diffraction from a planar screen with complex amplitude transmittance $\hat{f}(x)$. The complex amplitude distribution $\hat{g}(x)$ of light in a diffraction plane at distance $d$ is given by the Fresnel integral (Equation 84):

$$
\begin{aligned}
\hat{f}_{\text {out }}(x) & =\int \hat{h}\left(x, x^{\prime}\right) \hat{f}_{\text {in }}\left(x^{\prime}\right) d x^{\prime} \\
\hat{h}_{\text {space }}\left(x, x^{\prime}\right) & =K_{\text {space }} \exp \left[i \pi\left(x-x^{\prime}\right)^{2} / \lambda d \mid,\right.
\end{aligned}
$$

assuming illumination of the screen by a uniform plane wave. Now, with $f(x / s) \equiv \hat{f}(x)$, it is possible to cast this integral in the form of the integral of Equation 92 by identifying $\phi=a \pi / 2=\arctan \left(\lambda d / s^{2}\right), M=\sqrt{1+(\lambda d / s)^{2}}$, $\lambda R=\left(s^{4}+\lambda^{2} d^{2}\right) / \lambda d$. (The same results can be arrived at by comparing Equation 96 with Equation 88, or by specializing Equations 97, 98, and 99 to $A=D=1, B=\lambda d, C=0$.)

At a distance $d$ from the diffracting object or aperture, we observe the $a$ th order fractional Fourier transform of the object on a spherical reference surface with radius $R$. The transform is magnified by $M$. As $d$ is increased 
from 0 to $\infty$, the order $a$ of the fractional transform increases according to $a=(2 / \pi) \arctan \left(\lambda d / s^{2}\right)$ from 0 to 1 (Fig. 7). Letting $d \rightarrow \infty$, we obtain $a=1$, $M=\lambda d / s^{2} \propto d$, and $R=d$, which we readily associate with the Fourier diffraction pattern, which is nothing but the Fourier transform of the diffracting screen. Note that in this limit, the magnification and radius of curvature are both proportional to the distance $d$.

Thus we see that the propagation of light along the $+z$ direction can be viewed as a process of continual fractional Fourier transformation. As light propagates, its distribution evolves through fractional transforms of increasing orders. The fact that the far-field diffraction pattern is the Fourier transform of the diffracting object is one of the central results of diffraction theory. We have shown that the field at closer distances is given by fractional Fourier transforms of the diffracting object.

More generally, there exists a fractional Fourier transform relation between the amplitude distribution of light on two spherical reference surfaces of given radii and separation [Ozaktas and Mendlovic, 1994, 1995]. It is possible to determine the order and scale parameters associated with this fractional transform given the radii and separation of the surfaces. Alternatively, given the desired order and scale parameters, it is possible to determine the necessary radii and separation.

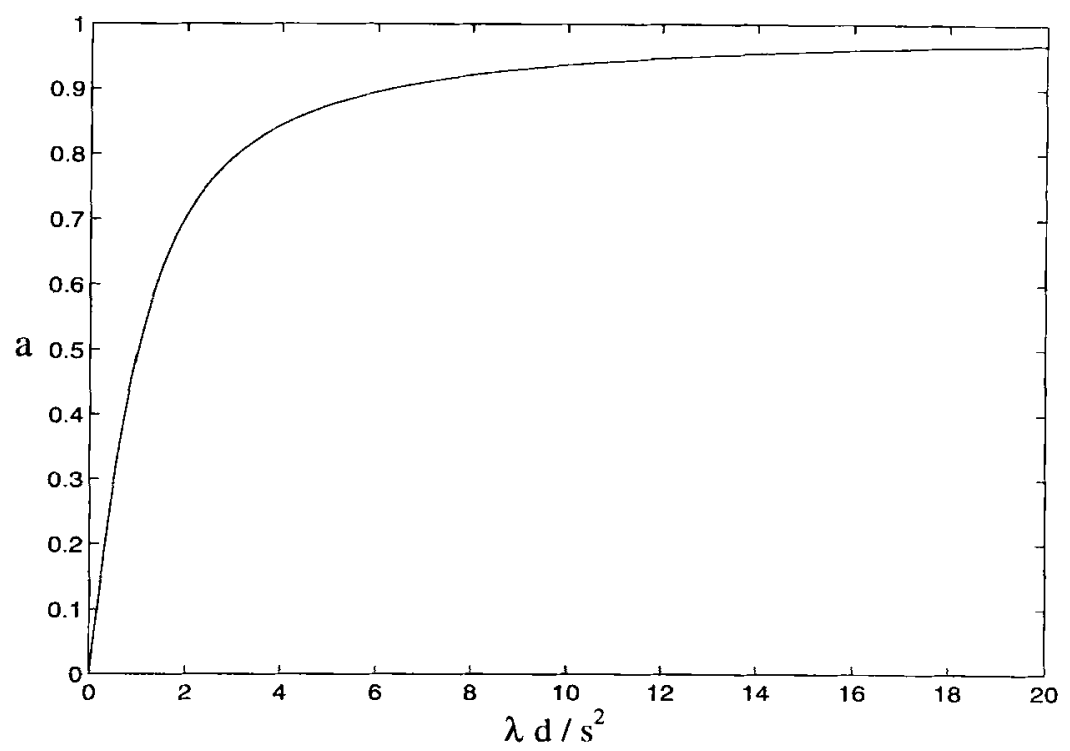

FIGURE 7. $a=(2 / \pi) \arctan \left(\lambda d / s^{2}\right)$ as a function of $\lambda d / s^{2}$. 


\section{E. Fourier Optical Systems}

We will now see that any quadratic-phase system can always be interpreted as a fractional Fourier transforming system. Thus the fractional Fourier transform can describe all systems composed of an arbitrary number of lenses separated by arbitrary distances, whereas imaging and Fourier transforming systems are only special cases.

All of the essential ingredients have already been provided and it merely remains to state the result. Any quadratic-phase system (Equation 82) can be characterized by its $A B C D$ parameters. As discussed earlier, the kernel in Equation 82 can be cast in the form of the kernel in Equation 92 by identifying $\phi=a \pi / 2, M$, and $R$ according to Equations 97,98 , and 99 . Since any quadratic-phase system can be interpreted as a fractional Fourier transform, and since any optical system consisting of an arbitrary concatenation of lenses and sections of free-space can be modeled as a quadraticphase system, it follows that such an optical system can also be interpreted in terms of the fractional Fourier transform.

A concrete example will be useful. Figure 8a shows a system consisting of several lenses whose focal lengths have been indicated in meters. The input plane is taken as $z=0$. The output plane is variable, ranging from $z=0$ to $z=2 \mathrm{~m}$. Two rays have been drawn through the system. Let $A(z), B(z), C(z)$, $D(z)$ denote the $A B C D$ parameters of the section of the system occupying the interval $[0, z]$, which can be readily calculated using the matrices for lenses and sections of free-space and the concatenation property. Also let $[x(z) p(z)]^{\mathrm{T}}$ denote the ray vector at $z$. Then,

$$
\left[\begin{array}{l}
x(z) \\
p(z)
\end{array}\right]=\left[\begin{array}{ll}
A(z) & B(z) \\
C(z) & D(z)
\end{array}\right]\left[\begin{array}{l}
x(0) \\
p(0)
\end{array}\right] .
$$

We further let $\phi(z)=a(z) \pi / 2, M(z) . R(z)$ represent the order, magnification, and phase curvature of the fractional Fourier transform observed at $z$. These can be determined again by using Equations 97,98 , and 99 .

The fractional transform order $a(z)$, the scale parameter $M(z)$, and the radius $R(z)$ of the spherical surface on which the perfect transform is observed are plotted as functions of $z$ in Fig. 8 [Ozaktas and Erden, 1997]. Letting $j$ denote an arbitrary integer, when $a=4 j$ we observe an erect image, when $a=4 j+2$ we observe an inverted image, when $a=4 j+1$ we observe the common Fourier transform, and when $a=4 j-1$ we observe an inverted Fourier transform (which is the same as an inverse Fourier transform). The reader should study the behavior of the two rays in conjunction with the graphs in Fig. 8. At $z=0.4$ we obtain a conventional Fourier transform $(a=1)$ as a result of the conventional $2 \mathrm{f}$ system 
(a.)

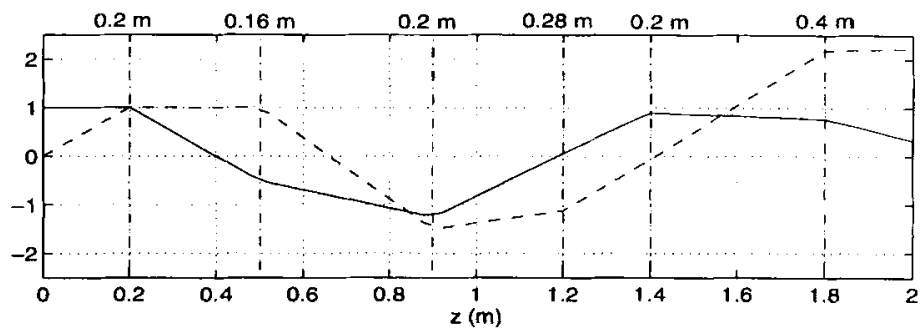

(b.)

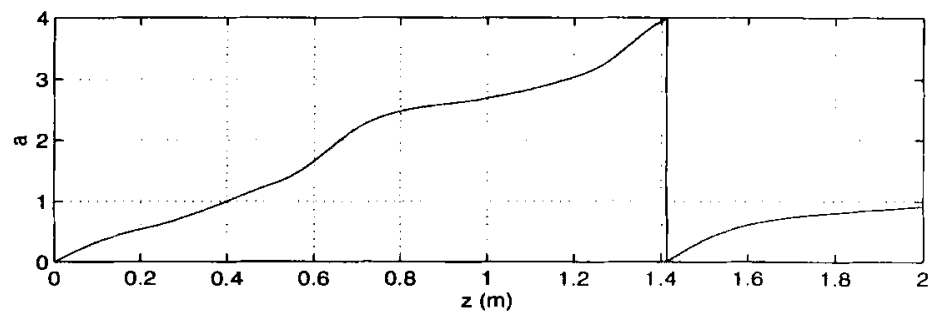

(c.)

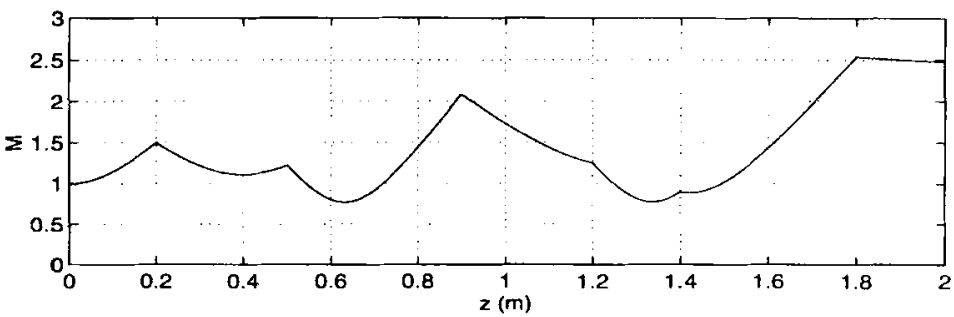

(d.)

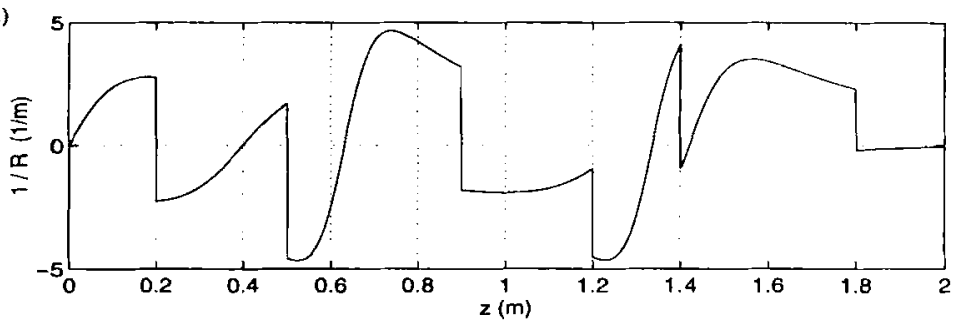

FIgure 8. Evolution of $a(z), M(z), 1 / R(z)$ as functions of $z$ [Reprinted from Optics Communications, 143, 75-83, 1997 with kind permission from Elsevier Science-NL, Sara Burgerhardtstraat 25, $1055 \mathrm{KV}$ Amsterdam, The Netherlands.].

occupying the interval $[0,0.4]$. An inverted image $(a=2)$ is observed at $z \approx 0.65$. We see that $M<1$ and $R>0$, as confirmed by an examination of the rays. (The ray represented by the solid line crosses the $z=0.65$ plane at a negative value (implying an inverted image) smaller than unity in magnitude (implying $M<1$ ), with a slope indicating divergence (implying 
$R>0$ ). An inverted Fourier transform $(a=3)$ is observed at $z \approx 1.2$, almost coincident with the lens at that location. An erect image $(a=1)$ is observed at $z \approx 1.4$, immediately after the lens at that location. The field curvature $1 / R$ of this image has a very small negative value and the magnification $M$ is slightly smaller than 1 . The imaging systems discussed in Bernardo and Soares [1994b] provide additional useful examples which the reader may wish to study in a similar manner [Ozaktas and Erden, 1997].

Fourier optical systems consist of an arbitrary number of thin filters sandwiched between arbitrary quadratic-phase systems. It readily follows that any Fourier optical system can be modeled as filters sandwiched between fractional Fourier transforms stages, or as repeated filtering in consecutive fractional Fourier domains (see Section XIII) [Ozaktas and Mendlovic, 1996].

\section{F. Optical Implementation of the Fractional Fourier Transform}

Here we mention a number of systems which map $\hat{f}_{\text {in }}(x)=f(x / s)$ into $\hat{f}_{\text {out }}(x) \propto f_{a}(x / s)$. Conceptually simplest is to use a section of quadratic graded-index media of length $d=\eta(a \pi / 2)=a d_{0}$ with $s^{2}=\lambda \eta / \eta_{0}$ (Subsection C).

In practice, systems consisting of bulk lenses may be preferred. Two such systems were first presented by Lohmann [1993]. We present these systems without derivation, referring the reader to Lohmann [1993] and Ozaktas and Mendlovic [1995] for details. The first system consists of a section of free-space of length $d$ followed by a lens of focal length $f$ followed by a second section of free-space of length $d$. To obtain an ath order fractional Fourier transform with scale parameter $s$, we must choose $d$ and $f$ according to

$$
\begin{aligned}
& d=\frac{s^{2}}{\lambda} \tan (\phi / 2), \\
& f=\frac{s^{2}}{\lambda} \csc \phi .
\end{aligned}
$$

The second system consists of a lens of focal length $f$ followed by a section of free-space of length $d$ followed by a second lens of focal length $f$. This time $d$ and $f$ must be chosen according to

$$
d=\frac{s^{2}}{\lambda} \sin \phi
$$




$$
f=\frac{s^{2}}{\lambda} \cot (\phi / 2)
$$

More general systems can easily be obtained by using the general formulation presented in Ozaktas and Mendlovic [1995], Mendlovic and others [1995b,c], Jiang [1995], Liu and others [1995], Sahin, Ozaktas, and Mendlovic [1995], and Ozaktas and Erden [1997].

\section{G. Gaussian Beam Propagation}

We have already seen that the propagation of light can be viewed as a process of continuous fractional Fourier transformation. In this subsection we will discuss the same facts, but this time in terms of Hermite-Gaussian beam expansions rather than Fresnel integrals or plane wave expansions. We will further see that the order of the fractional Fourier transform is proportional to the Gouy phase shift accumulated by the beam as it propagates.

Let $\hat{f}(x, 0)$ denote the complex amplitude distribution at the plane $z=0$. We can expand this function in terms of the Hermite-Gaussian functions:

$$
\begin{aligned}
\hat{f}(x, 0) & =\sum_{n=0}^{\infty} \hat{C}_{n} \sqrt{\frac{1}{s}} \psi_{n}\left(\frac{x}{s}\right), \\
\hat{C}_{n} & =\int \hat{f}(x, 0) \sqrt{\frac{1}{s}} \psi_{n}\left(\frac{x}{s}\right) d x .
\end{aligned}
$$

We can interpret the function $s^{-1 / 2} \psi_{n}(x / s)$ as the amplitude distribution of a one-dimensional $n$th order Hermite-Gaussian beam at its waist. Then, it becomes an easy matter to write the amplitude distribution $\hat{f}(x, z)$ at an arbitrary plane, since we know how each of the Hermite-Gaussian components propagates [Saleh and Teich, 1991]:

$\hat{f}(x, z)=\sum_{n=0}^{\infty} \hat{C}_{n} \frac{1}{m(z) s} \psi_{n}\left(\frac{x}{m(z) s}\right) \exp \left[i k z+\frac{i k x^{2}}{2 r(z)}-i(n+1 / 2) \zeta(z)\right]$.

In this equation $m(z) \equiv \sqrt{\pi} w(z) / s$, where $w(z)=w(0)\left[1+\left(z / z_{0}\right)\right]^{1 / 2}$ is the beam radius. Thus $m(0)=\sqrt{\pi} w(0) / s$, where $w(0)$ is the waist radius. The Rayleigh range $z_{0}$ is related to $s$ by the relation $s^{2}=\lambda z_{0}$. We also have $k=2 \pi / \lambda$, where $\lambda$ is the wavelength. Thus $r(z)=z\left[1+\left(z_{0} / z\right)^{2}\right]$ is the radius of curvature of the wavefronts, and $\zeta(z)=\operatorname{arctant}\left(z / z_{0}\right)$ is the Gouy phase shift [Saleh and Teich, 1991]. 
Equation 108 can be written in a considerably simple manner in terms of the fractional Fourier transform. Let us define functions with normalized arguments such that $\hat{f}(x, z)=f(x / s, z / s)$, etc. Then the amplitude distribution at any plane is given by

$$
\hat{f}(x, z)=\frac{1}{m(z)} e^{[i k z-i \zeta(z) / 2]} e^{\left[i k x^{2} / 2 r(z)\right]}\left(\mathscr{F}^{a(z)} f(a, 0)\right)\left(\frac{x}{m(z) S}\right)
$$

where

$$
a(z)=\frac{2}{\pi} \zeta(z)
$$

In Equation 109, the fractional Fourier transform is taken with respect to $u$, and $f(u, 0)=\hat{f}(s u, 0)$. Rewriting

$$
\phi(z)=\frac{\pi}{2} a(z)=\zeta(z),
$$

we see that the "angular order" $\phi$ of the fractional Fourier transform in question is simply equal to the Gouy phase shift accumulated in propagating from $z=0$ to $z$. As $z \rightarrow \infty$, we see that $\zeta(z) \rightarrow \pi / 2$ and $a(z) \rightarrow 1$, corresponding to the ordinary Fourier transform. This is the same result discussed in Subsection D.

This result can be generalized for propagation between two spherical references surfaces with arbitrary radii [Ozaktas and Mendlovic, 1994]. Let the radius of the surface at $z=z_{1}$ be denoted by $R_{1}$ and that of the surface at $z=z_{2}$ be denoted by $R_{2}$. The radii are positive if the surface is convex to the right. Then, there exists a fractional Fourier transform between these two surfaces whose order is given by

$$
a=\frac{\zeta\left(z_{2}\right)-\zeta\left(z_{1}\right)}{\pi / 2} .
$$

It is well known that if a certain relation between $R_{1}, R_{2}$ and $z_{2}-z_{1}$ holds, one obtains an ordinary Fourier transform relation between two spherical surfaces. What we have shown is that, for other values of the parameters, we obtain a fractional Fourier transform relation. Given any two spherical surfaces, what we need to do to find the order $a$ of the fractional Fourier transform relation existing between them is to find the Rayleigh range and waist location of a Gaussian beam that would "fit" into these surfaces, and then calculate $a$ from Equation 112.

We may also think of a complex amplitude distribution "riding" on a Gaussian beam wavefront. The spatial dependence of the wavefront as the 
wave propagates is like a carrier defining spherical surfaces, on top of which the complex amplitude distribution rides, being fractional Fourier transformed in the process.

Since laser resonators commonly consist of two spherical mirrors, it becomes possible to characterize such resonators in terms of a fractional order parameter, again obtained from Equation 112. The well-known stability (or confinement) condition for spherical mirror resonators can be stated in a particularly simple form in terms of the parameter $a$ : As long as $a$ is real, we have a stable resonator. (In our discussion we have implicitly assumed that $a$ and the Rayleigh range $z_{0}$ are real, which means that we have implicitly assumed stable resonators.) Unstable resonators are described by values of $a$ which are not real. Further details may be found in Ozaktas and Mendlovic [1994].

In addition to the relation between the fractional order parameter $\phi(z)$ and the Gouy phase shift $\zeta(z)$, the reader might also have noticed the similarity between the behavior of $M(z), R(z)$, and the common parameters of Gaussian beams, namely the beam diameter $w(z)$ and the wavefront radius of curvature $r(z)$. Indeed, readers well familiar with the propagation of Gaussian beams will have no difficulty interpreting the evolution of $R(z)$ and $M(z)$ in Fig. 8 as the wavefront radius and diameter of a Gaussian beam.

In considering systems such as that in Fig. 8, we will use $\zeta(z)$ to denote the accumulated Gouy phase shift with respect to the input plane at $z=0$, rather than the conventional Gouy phase shift with respect to the last waist of the beam [Erden and Ozaktas, 1997]. Essentially, the accumulated Gouy phase shift of a Gaussian beam passing through an optical system is defined as the phase accumulated by the beam in excess of the phase accumulated by a plane wave passing through the same system.

In Ozaktas and Erden [1997] we have determined how the expressions for $\zeta(z), w(z)$, and $r(z)$ are related to the expressions for $\phi(z), M(z)$, and $R(z)$ (given in Equations 97, 98, and 99). The main result can be stated as follows: "Let the output of an arbitrary system consisting of lenses and sections of free space be interpreted as a fractional Fourier transform of the input of order $\phi(z)$ with scale factor $M(z)$ observed on a spherical surface of radius $R(z)$. Let a Gaussian beam whose waist is located at $z=0$ with waist diameter $w_{0}$ exhibit an accumulated Gouy phase shift $\zeta(z)$, beam diameter $w(z)$, and wavefront radius of curvature $r(z)$ at the output of the same system. If the unit $s$ appearing in Equations 92 and 96 is related to $w_{0}$ as $s=\sqrt{\pi} w_{0}$, then $\phi(z)=\zeta(z), M(z)=w(z) / w_{0}$, and $R(z)=r(z) . "$

The reader is referred to Ozaktas and Erden [1997] for further details. 


\section{Applications to Signal and Image Processing}

The fractional Fourier transform has found many applications in optical and digital signal and image processing, where the ordinary Fourier transform has traditionally played an important role. Here we satisfy ourselves by considering a number of basic concepts and simple application examples.

In many signal processing applications, signals which we wish to recover are degraded by a known distortion and/or by noise. Then the problem is to reduce or eliminate these degradations. Appropriate solutions to such problems depend on the observation model and the objectives as well as the prior knowledge available about the desired signal, degradation process, and noise. A commonly used observation model is

$$
f_{\mathrm{obs}}(u)=\int h\left(u, u^{\prime}\right) f\left(u^{\prime}\right) d u^{\prime}+n(u)
$$

where $h\left(u, u^{\prime}\right)$ is the kernel of the linear system that degrades the desired signal $f(u)$, and $n(u)$ is an additive noise term. The problem is to find an estimation operator represented by the kernel $g\left(u, u^{\prime}\right)$, such that the estimated signal

$$
f_{\mathrm{est}}(u)=\int g\left(u, u^{\prime}\right) f_{\mathrm{obs}}\left(u^{\prime}\right) d u^{\prime}
$$

minimizes the mean square error defined as

$$
\sigma_{e}^{2}=\overline{\int\left|f_{\text {est }}(u)-f(u)\right|^{2} d u},
$$

where the overline denotes an ensemble average. The classical Wiener filter provides a solution to the preceding problem when the degradation is time-invariant and the input and noise processes are stationary. The Wiener filter is time-invariant and can thus be expressed as a convolution and implemented effectively with a multiplicative filter in the conventional Fourier domain with the fast Fourier transform algorithm (Fig. 9a). For an arbitrary degradation model or nonstationary processes, the classical Wiener filter often cannot provide a satisfactory result. In this case the optimum recovery operator is in general time-varying and has no fast implementation.

The dual of filtering in the ordinary Fourier domain is filtering in the space- or time-domain (Fig. 9b). This operation simply corresponds to multiplying the original function with a mask function. Filtering in the 

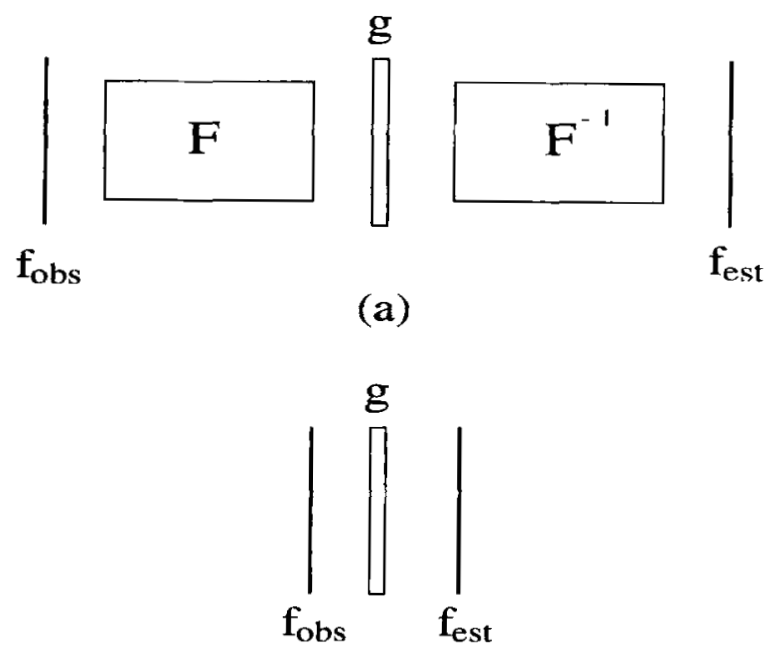

(b)
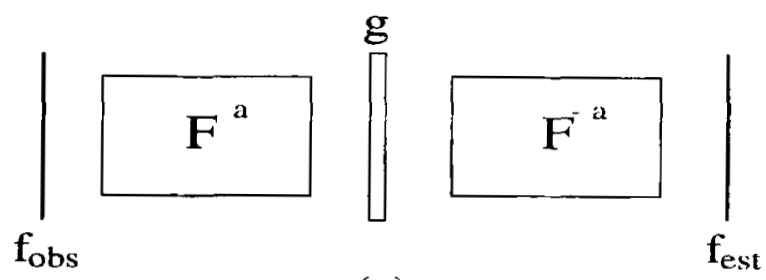

(c)

Flgure 9. (a) Filtering in the Fourier domain. (b) Filtering in the space (or time) domain. (c) Filtering in the $a$ th order fractional Fourier domain.

ordinary space or Fourier domains can be generalized to filtering in the ath order fractional Fourier domain (Fig. 9c) [Mendlovic and others, 1996b; Ozaktas, 1996; Zalevsky and Mendlovic, 1996; Kutay and others, 1997]. For $a=1$ this reduces to the ordinary multiplicative Fourier domain filter, and for $a=0$ it reduces to space-domain multiplicative filtering.

To understand the basic motivation for filtering in fractional Fourier domains, consider Fig. 10, where the Wigner distributions of a desired signal and an undesired distortion are superimposed. We observe that they overlap in both the 0th and 1st domains, but they do not overlap in the 0.5th domain (consider the projections onto the $u_{0}=u, u_{1}=u$, and $u_{0.5}$ axes). Although we cannot eliminate the distortions in the space or frequency domains, we can eliminate them easily by using a simple amplitude mask in the 0.5 th domain. 


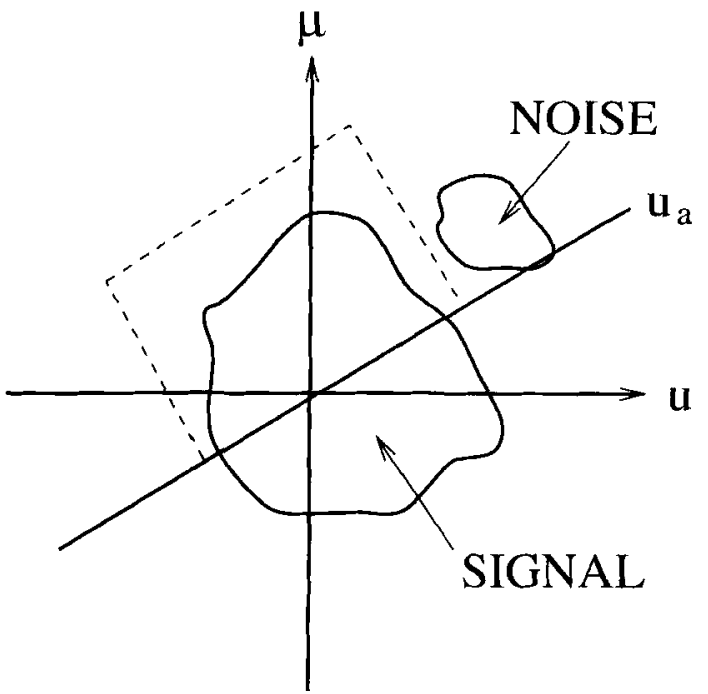

FIGURE 10. Filtering in fractional Fourier domains as observed in the space- (or time-) frequency plane.

We now discuss the optimal filtering problem mathematically. The estimated (filtered) signal $f_{\text {est }}$ is expressed as (Fig. 9c)

$$
\begin{aligned}
& f_{\text {est }}(u)=\mathscr{T}_{\text {single }} f_{\text {obs }}(u), \\
& \mathscr{T}_{\text {single }}=\mathscr{F}^{-a} \Lambda_{g} \mathscr{F}^{a},
\end{aligned}
$$

where $\mathscr{F}^{a}$ is the $a$ th order fractional Fourier transform operator, $\Lambda_{g}$ denotes the operator corresponding to multiplication by the filter function $g(u)$, and $\mathscr{T}_{\text {single }}$ is the operator representing the overall filtering configuration. According to Equation 117, we first take the ath order fractional Fourier transform of the observed signal $f_{\text {obs }}(u)$, then multiply the transformed signal with the filter $g(u)$ and take the inverse ath order fractional Fourier transform of the resulting signal to obtain our estimate. Since the fractional Fourier transform has efficient digital and optical implementations, the cost of fractional Fourier domain filtering is approximately the same as the cost of ordinary Fourier domain filtering. With the above form of the estimation operator, the problem is to find the optimum multiplicative filter function $g_{\text {opt }}(u)$ that minimizes the mean-square error defined in Equation 115.

For a given transform order $a, g_{\text {opt }}\left(u_{a}\right)$ can be found analytically using the orthogonality principle or the calculus of variations (Kutay and others, 
1997):

$$
g_{\mathrm{opt}}\left(u_{a}\right)=\frac{\iint K_{a}\left(u_{a}, u\right) K_{-a}\left(u_{a}, u^{\prime}\right) R_{f f_{\mathrm{obs}}}\left(u, u^{\prime}\right) d u^{\prime} d u}{\iint K_{a}\left(u_{a}, u\right) K_{-a}\left(u_{a}, u^{\prime}\right) R_{\int_{\mathrm{abs}} \int_{\mathrm{obs}}}\left(u, u^{\prime}\right) d u^{\prime} d u}
$$

where the stochastic auto- and cross-correlation functions $R_{f f_{\mathrm{obs}}}\left(u, u^{\prime}\right)$ and $R_{f_{\text {obs }} f_{\text {obs }}}\left(u, u^{\prime}\right)$ can be computed from the correlation functions $R_{f f}\left(u, u^{\prime}\right)$ and $R_{n n}\left(u, u^{\prime}\right)$ (which are assumed to be known).

Fractional Fourier domain filtering is particularly advantageous when the distortion or noise is of a chirped nature. Such situations are encountered in many real-life applications. For instance, a major problem in the reconstruction from holograms is the elimination of twin-image noise. Since this noise is essentially a modulated chirp signal, it can be dealt with by fractional Fourier domain filtering. Another example is the correction of the effects of point or line defects found on lenses or filters in optical systems, which appear at the output plane in the form of chirp artifacts. Another application arises in synthetic aperture radar which employs chirps as transmitted pulses, so that the measurements are related to the terrain reflectivity function through a chirp convolution. This process results in chirp-type disturbances caused by moving objects in the terrain, which should be removed if high-resolution imaging is to be achieved. Fractional Fourier domain filtering has also been applied to restoration of images blurred by camera motion or atmosphere turbulence [Kutay and Ozaktas, 1998].

Further generalizations of the concept of filtering in fractional Fourier domains have been referred to as multistage (repeated) and multichannel (parallel) filtering in fractional Fourier domains [Erden, 1997; Erden and others, 1997a,b; Ozaktas, Erden, and Kutay, 1997; Kutay and others, $1998 \mathrm{a}, \mathrm{b}]$. These systems consist of $M$ signal-stage fractional Fourier domain stages in series or in parallel (Fig. 11a, b). $M=1$ corresponds to single-stage filtering in both cases. In the multistage system shown in Figure 11a, the input is first transformed into the $a_{1}$ th domain, where it is multiplied by a filter $g_{1}(u)$. The result is then transformed back into the original domain and the same process is repeated $M$ times consecutively. (Note that this amounts to sequentially visiting the domains $a_{1}, a_{2}-a_{1}, a_{3}-a_{2}$, etc. and applying a filter in each.) On the other hand, the multichannel filter structure consists of $M$ single-stage blocks in parallel (Fig. 11b). For each channel $k$, the input is transformed to the $a_{k}$ th domain, multiplied with a filter $g_{k}(u)$, and then transformed back. Let $\Lambda_{g_{j}}$ denote the operator corresponding to multiplication by the filter function $g_{j}(u)$. Then, the outputs $f_{\text {est ser }}(u)$ and $f_{\text {est par }}(u)$ of the serial and parallel configurations are related to the input $f_{\text {obs }}(u)$ 

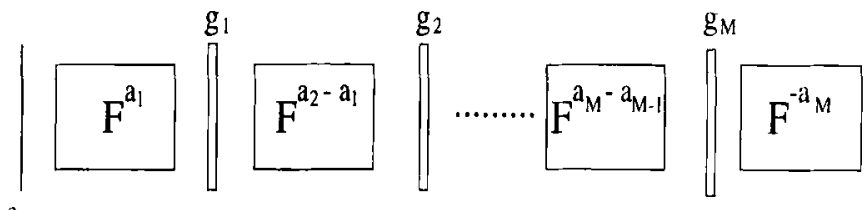

$\mathrm{f}_{\mathrm{obs}}$

(a)

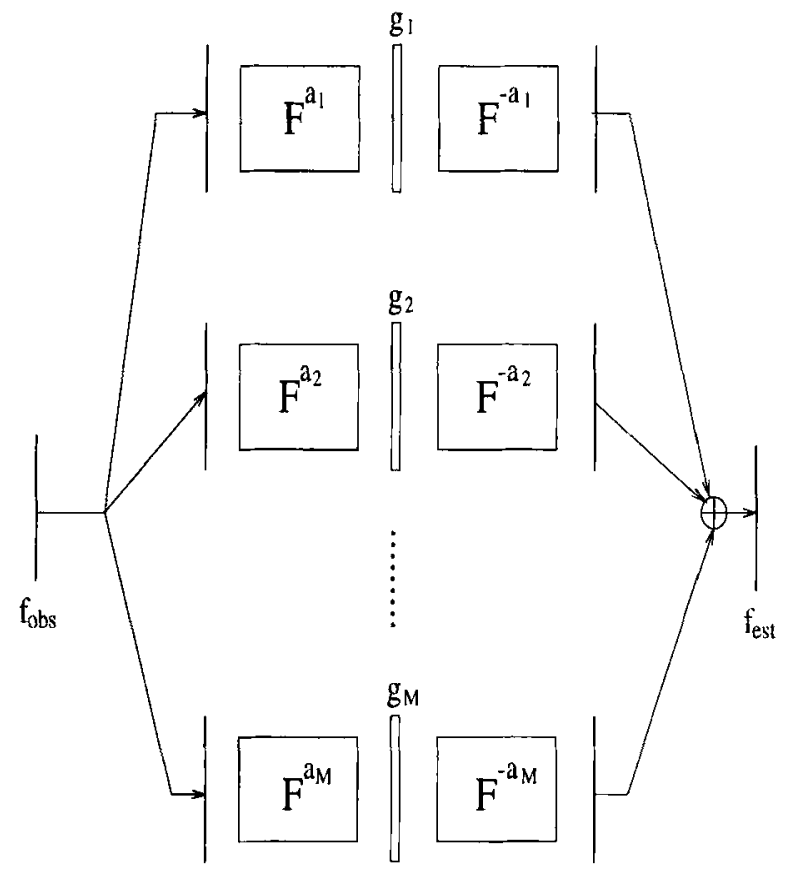

Figure 11. (a) Multistage filtering in fractional Fourier domains. (b) Multichannel filtering in fractional Fourier domains.

according to the relations

$$
\begin{aligned}
& f_{\text {est ser }}(u)=\left[\mathscr{F}^{-a_{M}} \Lambda_{g_{\mathcal{M}}} \ldots \mathscr{F}^{a_{2}-a_{1}} \Lambda_{g_{1}} \mathscr{\mathscr { F }}^{a_{1}}\right] f_{\text {obs }}(u)=\mathscr{T}_{\text {ser }} f_{\text {obs }}(u), \\
& f_{\text {est par }}(u)=\left[\sum_{k=1}^{M} \mathscr{F}^{-a_{k}} \Lambda_{g_{k}} \mathscr{F}^{a_{k}}\right] f_{\text {obs }}(u)=\mathscr{T}_{\text {par }} f_{\text {obs }}(u),
\end{aligned}
$$

where $\mathscr{F}^{a_{j}}$ represents the $a_{j}$ th order fractional Fourier transform operator and $\mathscr{T}_{\text {ser }}, \mathscr{T}_{\text {por }}$ the operators representing the overall filtering configurations.

As $M$ is increased, both the cost and flexibility of the systems increase. 
The digital implementation of these systems takes $O(M N \log N)$ time and their optical implementation requires an $M$-stage or $M$-channel optical system, each of whose stages or channels should have space-bandwidth product $N$. The increase in flexibility as $M$ increases will often translate into a reduction of the estimation error. Thus we can trade off between cost and accuracy by choosing an appropriate number of stages or channels.

As a simple example, we consider restoration of images blurred by a

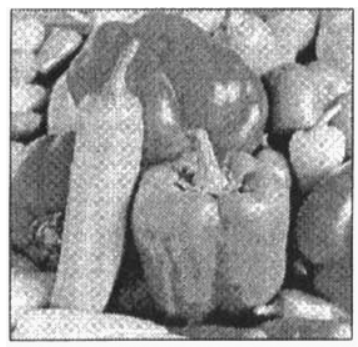

(a)

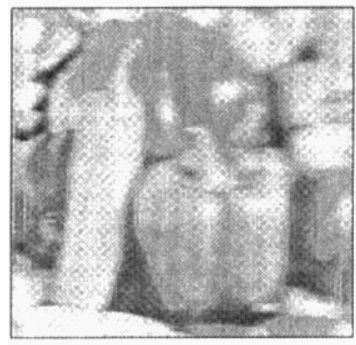

(c)

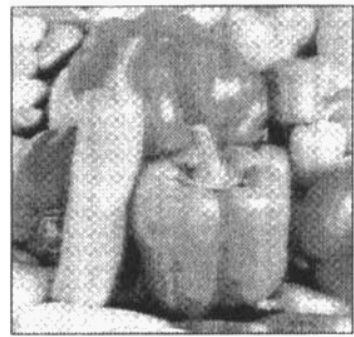

(e)

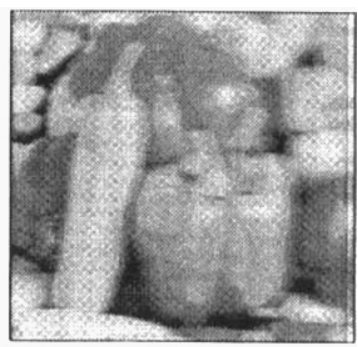

(b)

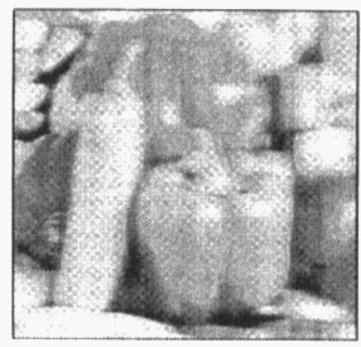

(d)

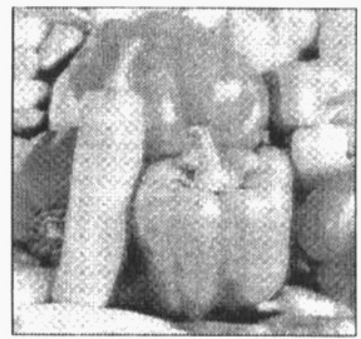

(f)

FIGURE 12. Image restoration with the fractional Fourier transform. 
nonconstant velocity (space-variant) moving camera. Figure 12a shows the original image, and Fig. 12b shows the blurred image. Figure $12 \mathrm{c}$ shows the restoration possible by using ordinary Fourier domain filtering, and Fig. $12 \mathrm{~d}$ shows restoration possible by single-stage filtering. In this case the optimal domain was $a=0.7$, resulting in a mean-square error of $5 \%$. Figure $12 \mathrm{e}$ and Fig. $12 \mathrm{f}$ show the restored images obtained by using multichannel and multistage filtering configurations with $M=5$. We see that the two latter options offer the best performance.

A further extension of these concepts is to combine the serial and parallel filtering configurations in an arbitrary manner to obtain generalized filtering configurations or circuits (Fig. 13) [Kutay and others, 1998a, b].

In the preceding discussion we have posed the multistage and multichannel configurations as filter structures for optimal image estimation. They can also be used for cost-efficient synthesis of desired linear systems, transform-

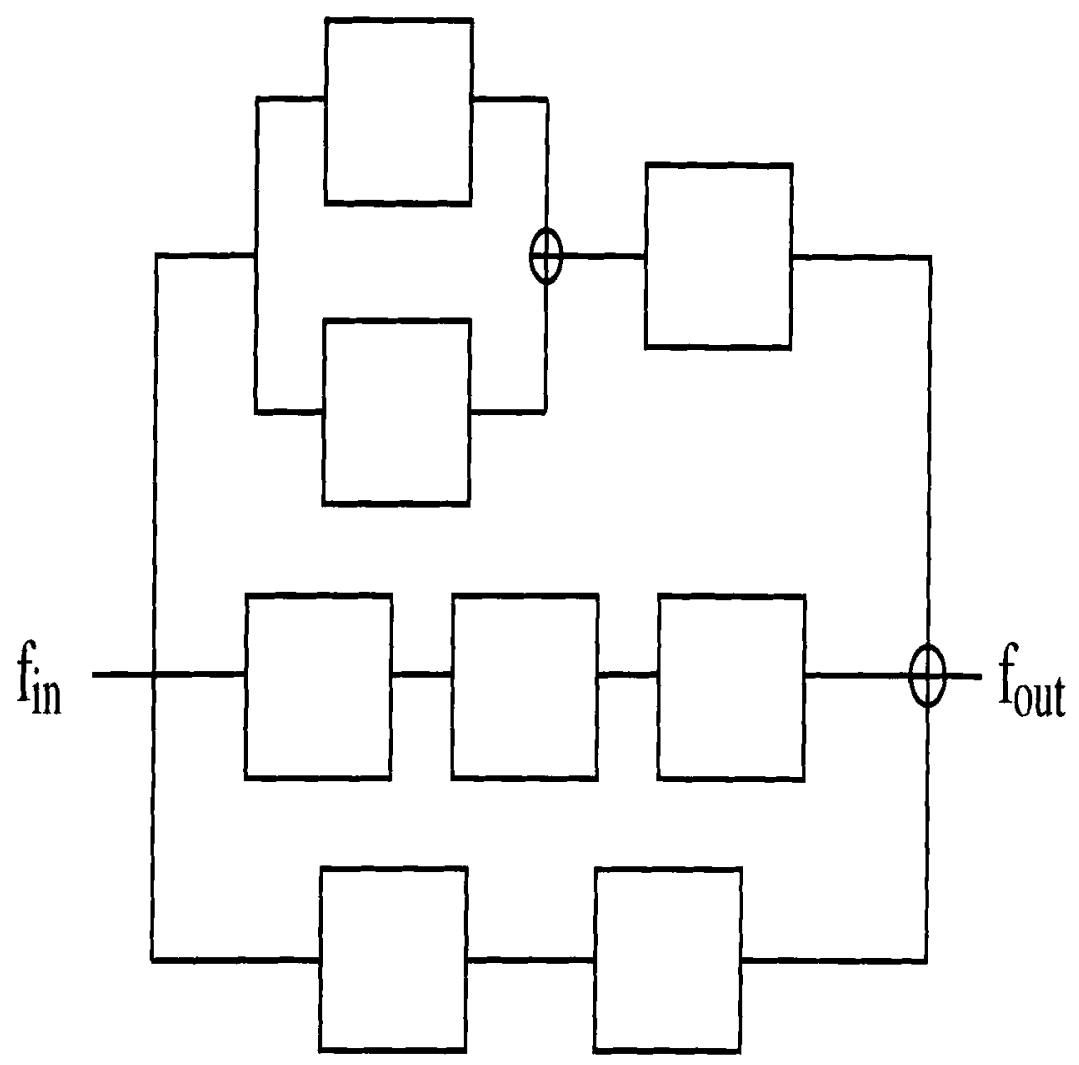

FIGURE 13. Filter circuits. Each block corresponds to single-stage filtering. 
ations, or mappings, including geometric distortion compensators, and beam shapers and synthesizers as well as linear recovery operators. In this approach, given a general linear system $\mathscr{H}$ characterized by the kernel $h(u$, $\left.u^{\prime}\right)$ which we wish to implement, we try to find the optimal orders $a_{k}$ and filter coefficients $g_{k}$ such that the overall linear operators $\mathscr{T}_{\text {single }}, \mathscr{T}_{\text {ser }}$, or $\mathscr{T}_{\text {par }}$ (as given by Equation 117, Equation 119, or Equation 120) is as close as possible to $\mathscr{H}$, according to some specified criteria (such as minimum Froebenius norm of the difference of the kernels).

The optical and digital implementations of general linear systems are costly. Using the abovementioned approach, it is possible to approximate the systems by multistage or multichannel filtering operations in fractional Fourier domains, which are much cheaper to implement. This would allow signficant savings in cost with little or no decrease in performance. Further discussion of this approach in a signal processing context may be found in Erden [1997], Erden and Ozaktas [1998], Ozaktas, Erden, and Kutay [1997], and Kutay and others [1998a]. We believe that this approach will find further applications in many other contexts.

Finally, we note that optimal filtering and image restoration is only one of the many signal processing applications explored. Correlation and pattern recognition applications have also received a considerable amount of interest. We refer the reader to the chapter by Mendlovic, Zalevsky, and Ozaktas [1998] and also to the following papers: Mendlovic, Ozaktas, and Lohmann [1995d]; Alieva and Agulló-López [1995]; García and others [1996]; Lohmann, Zalevsky, and Mendlovic [1996b]; Bitran and others [1996]; and Mendlovic and others [1995a].

\section{ACKNOWLEDGMENTS}

We acknowledge the contributions of M. Fatih Erden to various parts of this chapter. It is also a pleasure to acknowledge the benefit of interactions with Adolf W. Lohmann.

This chapter previously appeared as Ozaktas, Kutay, and Mendlovic 1998, parts of which previously appeared in Ozaktas and Mendlovic 1994, 1995, and Ozaktas and Erden 1997.

\section{REFERENCES}

Abe, S. and Sheridan, J. T. (1995a). Almost-Fourier and almost-Fresnel transformations. Optics Communications $113385-388$.

Abe, S. and Sheridan, J. T. (1995b). Comment on 'The fractional Fourier transform in optical propagation problems.' J. Modern Optics 42 2373-2378. 
Agarwal, G. S. and Simon, R. (1994). A simple realization of fractional Fourier transforms and relation to harmonic oscillator Green's function. Optics Commun. 110 23-26.

Alieva, T., Lopez, V, Agulló-López, F, and Almeida, L. B. (1994). The fractional Fourier transform in optical propagation problems. J. Modern Optics 41 1037-4044.

Alieva, T. and Agulló-López, F. (1995). Reconstruction of the optical correlation function in a quadratic refractive index medium. Optic's Communications $114161-169$. Erratum in 118657.

Almeida, L. B. (1994). The fractional Fourier transform and time-frequency representations. IEEE Trans. Signal Process. 42 3084-3091.

Alonso, M. A. and Forbes, G. W. (1997). Uniform asymptotic expansions for wave propagators via fractional transformations. Submitted.

Aytür, O. and Ozaktas, H. M. (1995). Non-orthogonal domains in phase space of quantum optics and their relation to fractional Fourier transforms. Optics Communications 120 $166-170$.

Bargmann, V. (1961). On a Hilbert space of analytic functions and an associated integra] transform. Part I. Comm. Pure and Applied Mathematics 11 187-214.

Bastiaans, M. J. (1978). The Wigner distribution applied to optical signals and systems. Optics Communications 25 26-30.

Bastiaans, M. J. (1979a). The Wigner distribution function and Hamilton's characteristics of an geometric-optical system. Optics Communications $30321-326$.

Bastiaans, M. J. (1979b). Wigner distribution function and its application to first-order optics. J. Optical Society of America A 69 1710-1716.

Bastiaans, M. J. (1989). Propagation laws for the second-order moments of the Wigner distribution function in first-order optical systems. Optik 82 173-181.

Bastiaans, M. J. (1991). Second-order moments of the Wigner distribution function in first-order optical systems. Optik 88 163-168.

Beck, M., Rayner, M. G., Walmsley, I. A., and Kong, V. (1993). Chronocyclic tomography for measuring the amplitude and phase structure of optical pulses. Optics Letters 18 2041-2043.

Bernardo, L. M. and Soares, O. D. D. (1994a). Fractional Fourier transforms and optical systems. Optics Communications 110 517-522.

Bernardo, L. M. and Soares, O. D. D. (1994b). Fractional Fourier transforms and imaging. $J$. Optical Society of America A 11 2622-2626.

Bitran, Y, Zalevsky, Z., Mendlovic, D., and Dorsch, R. G. (1996). Fractional correlation operation: Performance analysis. Applied Optics 35 297-303.

Bracewell, R. N. (1995). Two-Dimensional Imaging. Prentice-Hall, Englewood Cliffs, NJ. 1995.

Claasen, T. A. C. M. and Mecklenbräuker, W. F. G. (1980a). The Wigner distribution-a tool for time-frequency signal analysis. Part I: continuous-time signals. Philips $J$. Research 35 $217-250$.

Claasen, T. A. C. M. and Mecklenbräuker, W. F. G. (1980b). The Wigner distribution - a tool for time-frequency signal analysis. Part II: discrete-time signals. Philips $J$. Research 35 276-300.

Claasen, T. A. C. M. and Mecklenbräuker, W. F. G. (1880c). The Wigner distribution-a tool for time-frequency signal analysis. Part III: relations with other time-frequency signal transformations. Philips J. Research 35 372-389.

Cohen, L. (1989). Time-frequency distribution - a review. Proceedings of IEEE 77 941-981.

Cohen, L. (1995). Time-Frequency Analysis Prentice-Hall, Englewood Cliffs, NJ.

Condon, E. U. (1937). Immersion of the Fourier transform in a continuous group of functional transformations. Proc. National Academy of Sciences 23 158-164.

de Bruijn, N. G. (1973). A theory of generalized functions, with applications to Wigner distribution and Weyl correspondence. Nicuw Archief voor Wiskunde 21 205-280. 
Dorsch, R. G. (1995). Fractional Fourier transformer of variable order based on a modular lens system. Applied Optics 34 6016-6020.

Dorsch, R. G. and Lohmann, A. W. (1995). Fractional Fourier transform used for a lens design problem. Applied Optics 34 4111-4112.

Dragoman, D. (1996). Fractional Wigner distribution function. J. Optical Society of America A $13474-478$.

Erden, M. F. (1997). Repeated Filtering in Consecutive Fractional Fourier Domains. Ph.D. Thesis, Bilkent University, Ankara.

Erden, M. F., Kutay, M. A., and Ozaktas, H. M. (1997b). Repeated filtering in consecutive fractional Fourier domains and its application to signal restoration. Sub. to appear IEEE Trans. Signal Procesing, 1990.

Erden, M. F., Ozaktas, H. M. (1997). Accumulated Gony phase shift in Gaussian beam propagation through first-order optical systems. J. Optical Society of America B 142190 2194.

Erden, M. F. and Ozaktas, H. M. (1998). Synthesis of general linear systems with repeated filtering in consecutive fractional Fourier domains. To appear in J. Optical Society of America A, 1998 .

Erden, M. F., Ozaktas, H. M., and Mendlovic, D. (1996a). Propagation of mutual intensity expressed in terms of the fractional Fourier transform. J. Optical Society of America A 13 $1068-1071$.

Erden, M. F., Ozaktas, H. M., and Mendlovic, D. (1996b). Synthesis of mutual intensity distributions using the fractional Fourier transform. Optics Communications 125 288-301.

Erden, M. F., Ozaktas, H. M., Sahin, A., and Mendlovic, D. (1997a). Design of dynamically adjustable anamorphic fractional Fourier transformer. Optics Communications $13652-60$.

Fonollosa, J. R. and Nikias, C. L. (1994). A new positive time-frequency distribution. In Proc. 1994 Int. Conf. Acoustics, Speech, and Signal Processing. IEEE, NJ. IV 301-304.

Garcia, J., Mendlovic, D., Zalevsky, Z., and Lohmann, L. (1996). Space-variant simultaneous detection of several objects by the use of multiple anamorphic fractional-Fourier-transform filters. Applied Optics 35 3945-3952.

Gómez-Reino, C., Bao, C., and Pérez, M. V. (1996). GRIN optics, Fourier optics and optical connections. In 17th Congress of the International Commission for Optics: Optics for Science and New Technology, SPIE Proceedings 2778 128-131, SPIE, Bellingham, Washington, 1996.

Gori, F., Santarsiero, M., and Bagini, V. (1994). Fractional Fourier transform and Fresnel transform. Atti Foundaz Georgio Ronchi.

Granieri, S., Trabocchi, O., and Sicre, E. E. (1995). Fractional Fourier transform applied to spatial filtering in the Fresnel domain. Optics Communications 119 275-278.

Hlawatsch, F. and Boudreaux-Bartels, G. F. (1992). Linear and quadratic time-frequency signal representations. IEEE Signal Processing Magazine April 21-67.

Jiang, Z. (1995). Scaling laws and simultaneous optical implementation of various order fractional Fourier transforms. Optics Letters 20 2408-2410.

Kutay, M. A., Arikan, O., Candan, C., Güleryüz, Ö., Erden, M. F., Ozaktas, H. M. (1998b). Cost-efficient approximation of linear systems with multi-channel fractional Fourier domain filtering. Submitted to IEEE Signal Process. Lett.

Kutay, M. A., Erden, M. F., Ozaktas, H. M., Arikan, O., Güleryüz, Ö., and Candan, Ç. (1998a). Space-Bandwidth efficient realizations of linear systems. Optics Letters, 23 1069-1071.

Kutay, M. A. and Ozaktas, H. M. (1998). Optimal image restoration with the fractional Fourier transform. J. Optical Society of America A 15 825-834.

Kutay, M. A., Ozaktas, H. M., Arikan, O., and Onural, L. (1997). Optimal Filtering in Fractional Fourier Domains. IEEE Trans Signal Process 15 1129-1143. 
Liu, S., Xu, J., Zhang, Y., Chen, L., and Li, C. (1995). General optical implementation of fractional Fourier transforms. Optics Letters 20 1053-1055.

Lohmann, A. W. (1993). Image rotation, Wigner rotation, and the fractional order Fourier transform. J. Optical Society of America A 10 2181-2186.

Lohmann, A. W. (1995). A fake zoom lens for fractional Fourier experiments. Optics Communications $115437-443$.

Lohmann, A. W., Mendlovic, D., Zalevsky, Z., and Dorsch, R. G. (1996a). Some important fractional transformations for signal processing. Optics Communications 125 18-20.

Lohmann, A. W. and Soffer, B. H. (1994). Relationships between the Radon-Wigner and fractional Fourier transforms. J. Optical Society of America A 11 1798-1801.

Lohmann, A. W., Zalevsky, Z., and Mendlovic, D. (1996b). Synthesis of pattern recognition filters for fractional Fourier processing. Optics Communications 128 199-204.

McAlister, D. F., Beck, M., Clarke, L., Meyer, A., and Rayner, M. G. (1995). Optical phase-retrieval by phase-space tomography and fractional-order Fourier transforms. Optics Letters $201181-1183$.

McBride, A. C. and Kerr, F. H. (1987). On Namias's fractional Fourier transform. IMA J. Applied Mathematics 39 159-175.

Mecklenbrauker, W. F. G. (1993). The Wigner Distribution: Theory and Applications in Signal Processing. W. F. G. Mecklenbrauker, ed. Elsevier, Amsterdam.

Mendlovic, D., Bitran, Y., Dorsch, R. G., Ferreira, C., Garcia, J., and Ozaktaz, H. M. (1995b). Anamorphic fractional Fourier transform: optical implementation and applications. Applied Optic's 34 7451-7456.

Mendlovic, D., Bitran, Y., Dorsch, R. G., and Lohmann, A. W. (1995a). Optical fractional correlation: experimental results. Applied Optics 34 1665-1670.

Mendlovic, D., Dorsch, R. G., Lohmann, A. W., Zalevsky, Z., and Ferreira, C. (1996a). Optical illustration of a varied fractional Fourier-transform order and the Radon-Wigner display. Applied Optics 35 3925-3929.

Mendlovic, D. and Ozaktas, H. M. (1993). Fractional Fourier transformations and their optical implementation, I. J. Optical Society of America A 10 1875-1881.

Mendlovic, D., Ozaktas, H. M., and Lohmann, A. W. (1994a). Graded-index fibers, Wignerdistribution functions and the fractional Fourier transform. Applied Optics 33 6188-6193.

Mendlovic, D., Ozaktas, H. M., and Lohmann, A. W. (1995d). Fractional correlation. Applied Optics 34 303-309.

Mendlovic, D., Zalevsky, Z., Konforti, N., Dorsch, R. G., and Lohmann, A. W. (1995c). Incoherent fractional Fourier transform and its optical implementation. Applied Optics 34 $7615-7620$.

Mendlovic, D., Zalevsky, Z., Lohmann, A. W., and Dorsch, R. G. (1996b). Signal spatialfiltering using the localized fractional Fourier transform. Optics Communications 126 14-18.

Mendlovic, D., Zalevsky, Z., and Ozaktas, H. M. (1998). The applications of the fractional Fourier transform to optical pattern recognition. In Optical Pattern Recognition, Academic Press.

Milhovilovic, D. and Bracewell, R. N. (1991). Adaptive chirplet representation of signals on time-frequency plane. Electronics Letters 27 1159-1161.

Moshinsky, M. and Quesne, C. (1971). Linear canonical transformations and their unitary representations. J. Mathematics Physics 12 1772-1780.

Moshinsky, M., Seligman, T. H., and Wolf, K. B. (1972). Canonical transformations and the radical oscillator and Coulomb problem. J. Mathematics Physics 13 901-907.

Mustard, D. A. (1987a). Lie group imbeddings of the Fourier transform. School of Mathematics Preprint AM87/13. The University of New South Wales, Kensington, Australia

Mustard, D. A. (1987b). The fractional Fourier transform and a new uncertainty principle. 
School of Mathematics Preprint AM87/14. The University of New South Wales, Kensington, Australia.

Mustard, D. A. (1989). The fractional Fourier transform and the Wigner distribution. School of Mathematics Preprint AM89/6. The University of New South Wales, Kensington, Australia.

Mustard, D. A. (1991). Uncertainty principles invariant under the fractional Fourier transform. J. Australian Mathematical Society B 33 180-191.

Mustard, D. A. (1996). The fractional Fourier transform and the Wigner distribution. $J$. Australian Mathematical Society B 38 209-219.

Mustard, D. A. (1997). Fractional convolution. To appear in J. Australian Mathematical Society $B$.

Namias, V. (1980). The fractional order Fourier transform and its application to quantum mechanics. J. Inst. Maths Applics 25 241-265.

Nazarathy, M. and Shamir, J. (1982). First-order optics - a canonical operator representation: lossless systems. J. Opt. Soc. Am. 72 356-364.

Ozaktas, H. M. (1996). Repeated fractional Fourier domain filtering is equivalent to repeated time and frequency domain filtering. Signal Processing 54 81-84.

Ozaktas, H. M., Arikan, O., Kutay, M. A., and Bozdagi, G. (1996b). Digital computation of the fractional Fourier transform. IEEE Trans Signal Processing 44 2141-2150.

Ozaktas, H. M. and Aytür, O. (1995). Fractional Fourier domains. Signal Processing 46 $119-124$

Ozaktas, H. M., Barshan, B., Mendlovic, D., and Onural, L. (1994a). Convolution, filtering, and multiplexing in fractional Fourier domains and their relation to chirp and wavelet transforms. J. Optical Society of America A 11 547-559.

Ozaktas, H. M. and Erden, M. F. (1997). Relationships among ray optical, Gaussian beam, and fractional Fourier transform descriptions of first-order optical systems. Optics Communications $14375-86$.

Ozaktas, H. M., Erden, M. F., and Kutay, M. A. (1997). Cost-Efficient Approximation of Linear Systems with Repeated Filtering. Submitted to IEEE Signal Processing Lett.

Ozaktas, H. M., Erkaya, N., and Kutay, M. A. (1996a). Effect of fractional Fourier transformation on time-frequency distributions belonging to the Cohen class. IEEE Signal Processing Lett. $310-11$.

Ozaktas, H. M., Kutay, M. A., and Mendlovic, D. (1998). The fractional Fourier transform. Technical Report BU-CEIS Introduction to fractional Fourier transform 9802. Bilkent University, Department of Computer Engineering and Information Sciences, Bilkent, Ankara.

Ozaktas, H. M. and Mendlovic, D. (1993a). Fourier transforms of fractional order and their optical interpretation. Optics Communications 101 163-169.

Ozaktas, H. M. and Mendlovic, D. (1993b). Fractional Fourier transformations and their optical implementation. II. J. Optical Society of America A $102522-2531$.

Ozaktas, H. M. and Mendlovic, D. (1994). Fractional Fourier transform as a tool for analyzing beam propagation and spherical mirror resonators. Optics Letters 19 1678-1680.

Ozaktas, H. M. and Mendlovic, D. (1995). Fractional Fourier optics. J. Optical Society of America A $12743-751$.

Ozaktas, H. M. and Mendlovic, D. (1996). Every Fourier optical system is equivalent to consecutive fractional-Fourier-domain filtering. Applied Optics 35 3167-3170.

Pellat-Finet, P. (1994). Fresnel diffraction and the fractional-order Fourier transform. Optics Letters 19 1388-1390.

Pellat-Finet, P. and Bonnet, G. (1994). Fractional order Fourier transform and Fourier optics. Optics Communications 111 141-154.

Pellat-Finet P. (1995). Transfert du champ électromagnétique par diffraction et transformation de Fourier fractionnaire. C R Acad. Sci. Paris 320 91-97. 
Raymer, M. G., Beck, M., and McAlister, D. F. (1994a). Complex wave-field reconstruction using phase-space tomography. Physical Review Letters 72 1137-1140.

Raymer, M. G., Beck, M., and McAlister, D. (1994b). Spatial and temporal optical field reconstruction using phase-space tomography. In Quantum Optics VI. Springer, Berlin.

Sahin, A., Ozaktas, H. M., and Mendlovic, D. (1995). Optical implementation of the twodimensional fractional Fourier transform with different orders in the two dimensions. Optics Communications $120134-138$.

Saleh, B. E. A. and Teich, M. C. (1991). Fundamental of Photonics. Wiley, New York.

Seger, O. (1993). Model Building and Restoration with Applications in Confocal Microscopy, $\mathrm{Ph} . \mathrm{D}$. thesis, Linköping University, Sweden.

Smithey, D. T., Beck, M., Raymer, M. G., and Faridani, A. (1993). Measurement of the Wigner distribution and the density matrix of a light mode using optical homodyne tomography: application to squeezed states and the vacuum. Physical Review Letters 70 1244-1247.

Wiener, N. (1929). Hermitian Polynomials and Fourier Analysis. Journal of Mathematics Physics MIT $1870-73$.

Wolf, K. B. (1979). Construction and properties of canonical transforms. In Integral Transforms in Science and Engineering. Plenum Press, New York.

Wood, J. C. and Barry, D. T. (1994a). Tomographic time-frequency analysis and its application toward time-varying filtering and adaptive kernel design for multicomponent linear-FM signals. IEEE Trans Signal Processing 42 2094-2104.

Wood, J. C. and Barry, D. T. (1994b). Linear signal synthesis using the Radon-Wigner transform. IEEE Trans Signal Processing 42 2105 2111.

Yurke, B., Schleich, W., and Walls, D. F. (1990). Quantum superpositions generated by quantum nondemolition measurements. Physical Rev. A 42 1703-1711.

Zalevsky, Z. and Mendlovic, D. (1996). Fractional Wiener filter. Applied Optics 35 3930-3936.

\section{ACKNOWLEDGMENTS}

We acknowledge the contributions of M. Fatih Erden to various parts of this chapter. It is also a pleasure to acknowledge the benefit of interactions with Adolf W. Lohmann.

This chapter previously appeared as Ozaktas, Kutay, and Mendlovic 1998, parts of which previously appeared in Ozaktas and Mendlovic 1994, 1995, and Ozaktas and Erden 1997. 